DIW BERLIN

Discussion

Papers
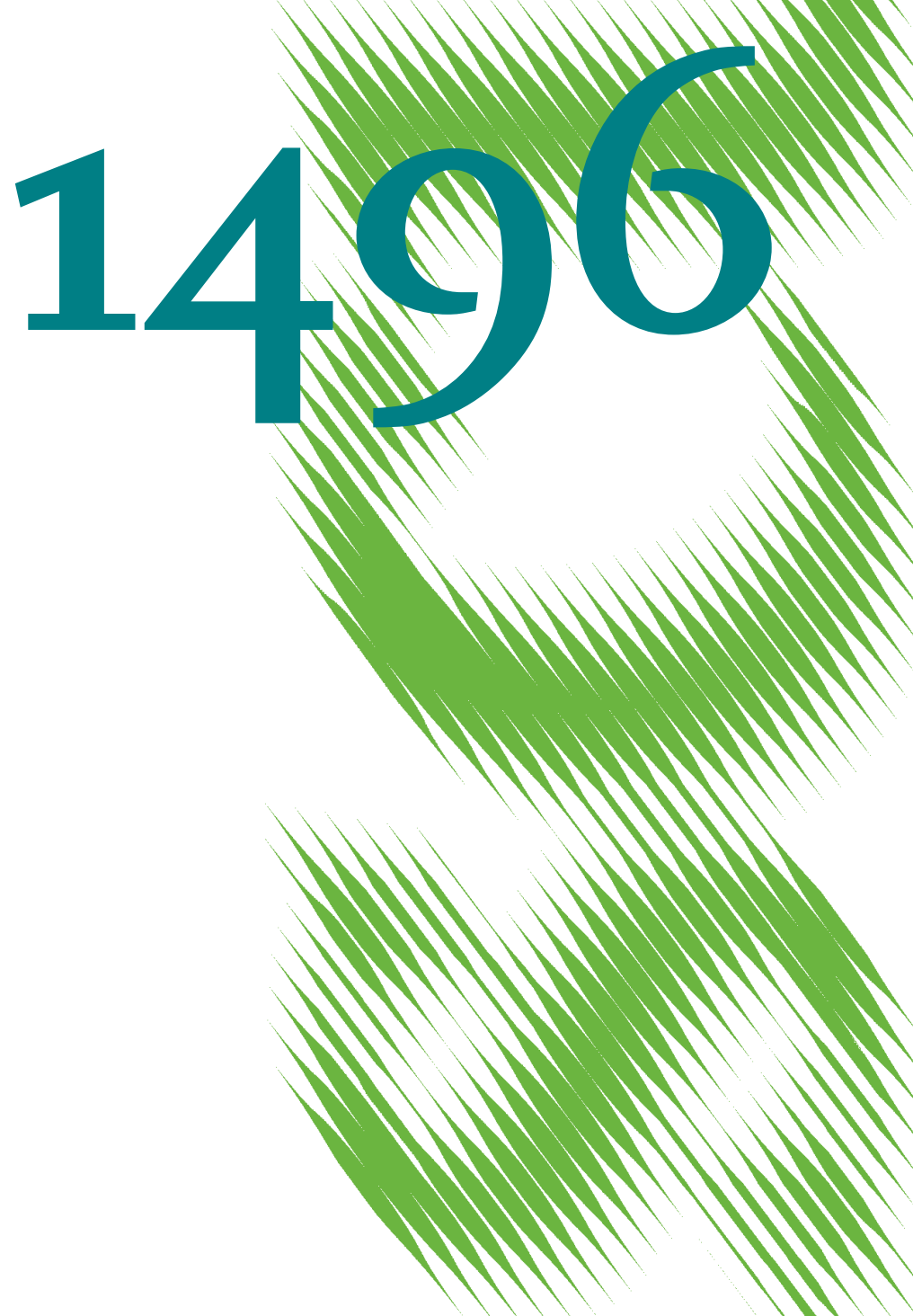

The Real-Time Predictive Content of Asset Price Bubbles for Macro Forecasts 
Opinions expressed in this paper are those of the author(s) and do not necessarily reflect views of the institute.

IMPRESSUM

(C) DIW Berlin, 2015

DIW Berlin

German Institute for Economic Research

Mohrenstr. 58

10117 Berlin

Tel. +49 (30) $89789-0$

Fax +49 (30) $89789-200$

http://www.diw.de

ISSN electronic edition 1619-4535

Papers can be downloaded free of charge from the DIW Berlin website:

http://www.diw.de/discussionpapers

Discussion Papers of DIW Berlin are indexed in RePEc and SSRN:

http://ideas.repec.org/s/diw/diwwpp.html

http://www.ssrn.com/link/DIW-Berlin-German-Inst-Econ-Res.html 


\title{
The real-time predictive content of asset price bubbles for macro forecasts
}

\author{
Benjamin Beckers*
}

July 27,2015

\begin{abstract}
This paper contributes to the debate of whether central banks can "lean against the wind" of emerging stock or house price bubbles. Against this background, the paper evaluates if new advances in real-time bubble detection, as brought forward by Phillips et al. (2011), can timely detect bubble emergences and collapses. Building on simulations, the paper shows that the detection capabilities of all indicators are sensitive to their exact specifications and to the characteristics of the bubbles in the sample. Therefore, the paper suggests a combination approach of different bubble indicators which helps to account for the uncertainty around start and end dates of asset price bubbles. Additionally, the paper then investigates if the individual and combination indicators carry predictive content for inflation and output growth when the real-time availability of all variables is taken into account. It finds that a combination indicator is best suited to uncover the most common stock and house price bubbles in the U.S. and shows that this indicator improves output forecasts.
\end{abstract}

Keywords: Asset price bubbles, financial stability, leaning-against-the-wind, monetary policy, real-time forecasting, unit root monitoring test.

JEL Classification: C22; C53; E44; E47; G12

*DIW Berlin, Graduate Center, Mohrenstraße 58, 10117 Berlin, Germany, bbeckers@diw.de. I thank Kerstin Bernoth, Jörg Breitung, Helmut Herwartz, Helmut Lütkepohl and Christian Proaño for their advice. Further, I thank participants of the ISF 2015, Riverside; the IAAE Annual Meeting 2015, Thessaloniki; the Warsaw International Economic Meeting (WIEM) 2015, Warsaw; and the workshop "Empirical Macroeconomics" at Free University Berlin for helpful comments and suggestions. Financial support by the German Research Foundation (DFG) (Project title: "Macroeconomic fundamentals of asset prices - state dependence and implications for the conduct of monetary policy") is gratefully acknowledged. 


\section{Introduction}

Following the 'dot-com' crisis of the late 1990's and particularly the recent global financial crisis, the importance of asset price cycles for macroeconomic stability is on the agenda of academic researchers and policy makers alike. Furthermore, the fact that these asset price booms have been accompanied with ample levels of liquidity, has raised questions about the role of monetary policy in the propagation of asset price bubbles. Exemplary, former ECB president Jean-Claude Trichet considers this, "to be one of the most challenging issues facing a modern central bank at the beginning of the 21st century" (Trichet, 2005). The responses of central banks to the recent global financial crisis cutting interest rates to the zero lower bound and providing additional lines of liquidity have exacerbated these concerns further, so that monetary policy makers are now monitoring asset markets closely for signs of exuberance (Draghi, 2015).

Against this background, a more active role of monetary policy in combating asset price bubbles early in their development (coined as "leaning against the wind") has been called for by many observers. That is, in contrary to the current policy of only lowering interest rates immediately after a crash, central banks should respond symmetrically and cautiously increase rates as soon as an asset price bubble is identified. However, for such a policy to be implementable, it is first required that policy makers are able to identify emerging asset price bubbles in real-time. Second, asset price bubbles should also provide reliable signals for central banks' ultimate primary and secondary targets of price stability and output and/or employment near potential levels. If asset price bubbles do not signal risks for the real economy, there is no incentive to raise the policy rate and cut-off real economic growth 11

This paper will therefore connect existing, yet separate, lines of research to address these two open issues. First, it assesses whether recently suggested monitoring indicators can detect price bubbles in the S\&P 500 and the U.S. housing market in real-time. Adding to the methodology of detecting bubbles, a new combination approach is suggested to account for the large uncertainty around bubble emergence and collapse dates. Second, this paper investigates whether bubble indicators contain additional value for predicting U.S. output growth and inflation in a real-time setting.

So far, most policy makers argue that the two requirements stated above are likely not met, as the detection of asset price bubbles has been considered to be impossible - not only ex post but also in real-time (cf. Trichet, 2005 and Kohn, 2006). However, this view has been questioned following the seminal paper by Phillips et al. (2011) (PWY11, henceforth). Building on recursive right-side unit root tests on price and dividend series, the authors show

\footnotetext{
${ }^{1}$ Additionally, asset price bubbles must also be sensitive to changes in the interest rate and the long-term expected benefits of pricking a bubble should exceed the immediate costs of lower output growth. This discussion is, however, not within the scope of this paper.
} 
that real-time monitoring approaches are capable of detecting periods that display patterns typical for asset price bubbles. This work has been developed further by Homm \& Breitung (2012) and by Phillips et al. (2013) (PSY13, henceforth) who generalize the initial work of PWY11 and develop a monitoring procedure that is robust to multiple periodically collapsing bubbles. Yet, as PWY13 show, all tests differ in their detection ability depending on the number and the timing of bubbles in the sample under consideration. Therefore, all tests, a priori, may provide some complementary value. Thus, this paper follows a suggestion by Harvey et al. (2015) and evaluates combinations building aggregating the information contained in individual indicators to account for uncertainty around the timing of bubbles.

The first finding of this paper highlights that bubble detection depends crucially on the indicators' exact specification. Specifically, it matters whether individual price and dividend series or the price-to-dividend ratio are tested for explosive roots - an issue that is not discussed carefully in the literature. Further, depending on the number of bubbles in the sample and their location, either the PWY11 or the PSY13 indicator are more likely to provide an accurate signal. Combination indicators make use of these complementary strengths and, thus, form a promising tool to hedge against the uncertainty around bubble start and end points as signaled by individual indicators. In application it is found that common bubble episodes detected by at least half of the indicators describe the build-up of a stock price bubble prior to the 1987 crash, the dot-com bubble starting around 1996, and the house price bubble that began in the early 2000's.

The only known study investigating the predictive content of asset price bubble indicators for real economic variables is Assenmacher-Wesche \& Gerlach (2010) (AWG10, henceforth). Certainly, asset (and in particular stock) prices are long considered to be valuable predictors for real economic variables, as they are inherently forward-looking. Empirical evidence by Stock \& Watson (2001), however, strongly questions their usefulness as predictors $2^{2}$ Yet, in addition to their forward-looking character, asset price developments can have immediate causal effects on real economic outcomes that are particularly relevant when considering the importance of bubble periods. Two transmission channels prominently discussed in the literature are the balance sheet (or collateral), and the lending (as introduced by Holmstrom \& Tirole, 1997) channels. Both suggest that rising asset prices can alleviate credit constraints for firms and households, thereby stimulating investment, consumption and, ultimately, output ${ }^{3}$ During a bubble, prices are, however, predominantly driven by speculative motives.

\footnotetext{
${ }^{2}$ The authors find that stock prices provide little predictive ability for output growth and none for inflation compared to simple autoregressive forecasts. House prices are studied to a far lesser extent, mostly due to data limitations. Nonetheless, the available studies also suggest little predictive use.

${ }^{3}$ The balance sheet channel theorizes that rising asset prices increase the value of collateral that firms and households can put up to borrow new funds. The lending channel refers to the role of financial intermediaries in credit supply. Rising asset prices boost banks' equity, thus making it easier for them to provide credit. Naturally, the reverse holds for falling prices.
} 
This may increase investment in the respective asset class more than fundamentally justified, which implies an inefficient allocation of resources across the economy. When prices eventually crash the feedback loop described above reverses and, additionally, the physical capital stock and/or employment in the bubble sector is likely to be inefficiently high, binding valuable resources. Bubble periods can thus intensify regular business cycle movements up to the degree that the economy overheats with severe and long-lasting recessions following 4 Therefore, one can expect that information about emerging asset price bubbles can contain predictive content for forecasting output and inflation.

Against these theoretical considerations, and questioning the scope for an activist monetary policy, AWG10 find that bubble indicators do not provide valuable information for forecasting output and inflation beyond the information that is already contained in other standard predictor variables. Yet, the study suffers from two main shortcomings. First, it does not make use of the new indicators introduced above, only considering indicators based on price deviations from a one-sided HP-filtered trend. Second, the paper does not consider the real-time dimension of all variables included in the forecasting exercise. As stock prices are available in real-time, they might contain information not included in variables that are only available with a lag and that are subject to revisions.

Therefore, the second contribution of this paper is to reevaluate the predictive content of asset price bubbles for inflation and industrial production when including the state-of-the-art indicators and their combinations introduced above. The target variables are chosen to reflect a CB's mandate and help determine whether the first requirements for a leaning-against-thewind policy are fulfilled. Furthermore, this paper specifically takes into account the real-time availability of all predictors. Following AWG10, the forecast accuracy of a benchmark model, including useful predictors such as output growth, inflation, unemployment and interest rates as identified by Stock \& Watson (2001), is evaluated first and then contrasted against an augmented model including bubble indicators.

This paper finds that several asset price bubble indicators carry significant additional predictive content for output growth and inflation. Foremost, these are the indicator proposed by PWY13 and a combination indicator suggested in this paper. By this, the paper questions the findings of AWG10. The findings of this paper also indicate that considering the realtime dimension of all variables is crucial. Since stock prices and dividends are available in real-time, their predictive content is understated when ignoring publication lags and revisions of the real economic variables included in the model. In contrast, the predictive content of house price bubble indicators is exacerbated when ignoring the real-time dimension, as these

\footnotetext{
${ }^{4}$ The negative real effects are likely to be largest for credit financed asset bubbles (Borio \& Lowe, 2004). A natural extension of the present paper is, thus, to investigate the predictive content of credit "booms." This is not done here, as credit data is only available on the quarterly frequency, limiting the scope for forecast evaluations.
} 
variables are available with a lag of two months. Stock prices are particularly useful for horizons of up to 24 months, while house prices add to forecasts for even longer horizons.

The paper is structured as follows. Section 2 introduces the indicators used for detecting asset price bubbles and discusses different specifications considered in the literature and their advantages. Further, the combination indicators are defined and their use is motivated. Section 3 evaluates the finite sample power properties of all indicators and the suggested combinations in different bubble environments. Section 4 then presents the evidence for stock and house price bubbles in the U.S. and introduces the forecast models used for predicting inflation and industrial production. Furthermore, the real-time data set is presented. Results of the forecast exercise are then discussed in Section 5 . Section 6 concludes.

\section{Asset price bubbles: Real-time detection}

\subsection{Definition and testability of asset price bubbles}

An asset price bubble is commonly defined to occur if an asset's price deviates from (and typically exceeds) its fundamental value (FV) due to unjustified beliefs about the asset's market price in the future. The key question in detecting a bubble is thus to determine the asset's unobservable underlying FV. The most prominent formulation and starting point for testing for the existence of (rational) bubbles is derived from the present value theory of finance and begins with the asset pricing equation

$$
P_{t}=\frac{1}{1+r} E_{t}\left[P_{t+1}+D_{t+1}\right]
$$

where $P_{t}, D_{t}$ are the asset's market price in and dividend accrued over period $t$, respectively, and $r$ is the (here time-constant) discount rate ${ }^{5}$ Note that this equation assumes risk-neutral agents $\sqrt[6]{6}$ Applying forward substitution one can show that (1) allows for the inclusion of a bubble component $B_{t}$ that measures the deviation of the time $t$ market price $P_{t}$ from the $F V_{t}$

$$
\begin{aligned}
P_{t} & =\sum_{i=1}^{\infty}\left(\frac{1}{1+r}\right)^{i} E_{t}\left[D_{t+i}\right]+\lim _{i \rightarrow \infty}\left(\frac{1}{1+r}\right)^{i} E_{t}\left[P_{t+i}\right] \\
& =F V_{t}+B_{t},
\end{aligned}
$$

\footnotetext{
${ }_{5}^{5}$ As Himmelberg et al. (2005) show, the fundamental determinants of house prices depend on several additional factors, including (among others) property taxes, tax deductibility of mortgage interest, and maintenance or depreciation costs, which are assumed to be constant here.

${ }^{6}$ See Gürkaynak (2008) for a thorough derivation and discussion of the model's underlying assumption.
} 
where $B_{t}>0$, if the usual transversality condition $\lim _{i \rightarrow \infty}\left(\frac{1}{1+r}\right)^{i} E_{t}\left[P_{t+i}\right] \rightarrow 0$ does not hold $]^{7}$ Importantly, this implies that the bubble component $B_{t}$ must grow exponentially with $r$ for (1) to hold and for the bubble not to shrink to zero in present value or to outgrow the

economy, i.e. $B_{t}=\frac{1}{1+r} E_{t}\left[B_{t+1}\right]$. Under the standard assumption that dividends follow a random walk (with drift), this has direct testable implications. When dividends follow a unit root process but a bubble is present, i.e. $B_{t}>0$, the price series must contain an explosive root that can only come from the bubble process. In this sense, a bubble is detected if the price process decouples from the dividend process. If dividends grow exponentially, too, no inference on the existence of a bubble component is possible in this setting.

Nonetheless, there are important limitations to bubble testing. Most importantly, as Flood \& Hodrick (1990) highlight, every test for the existence of rational bubbles per se relies on a correct specification of the FV. Thus, in fact empirical tests address the joint null hypothesis of the absence of bubbles and a correctly specified economic model. Hence, the rejection of the hypothesis cannot answer the question if a bubble is present indeed, or if simply a poor economic model has been employed. Still, empirical tests for bubbles can serve as specification tests for an economic model. Furthermore, for policy makers it is potentially of interest to detect common characteristics of "speculative bubbles" or "periods of exuberance" that can possibly indicate instabilities in the financial system based on past evidence (cf. Trichet, 2005). For this, however, the real-time detectability of asset price bubbles is crucial. Here, monitoring approaches can provide valuable insights 8

\subsection{Real-time detection of explosive behavior}

The literature on testing for bubbles has long focused on ex post tests for the existence of bubbles over an entire historical dataset. A survey on this literature including variancebound, two-step specification and unit-root tests is found both in Flood \& Hodrick (1990) and Gürkaynak (2008). While the first two approaches suffer mostly from practical issues in their implementation and have not been pursued, integration tests that build on the insights developed in the previous section, are being revived following the seminal paper of PWY11. In this paper, the authors show that the original unit root tests of Diba \& Grossman (1988) can

\footnotetext{
${ }^{7}$ For this general setting, it is extensively documented that bubbles can emerge, even if only some of the strict assumptions of infinitely-lived, rational and risk-neutral agents as well as complete markets are loosened. See Camerer (1989) and Stiglitz (1990) for surveys on the early theoretical work on the existence of rational bubbles, which focused predominantly on overlapping-generations models, the introduction of asymmetric information and/or incomplete markets restricting the opportunities for arbitrageurs. More recently, the role of agents' incentives, non-standard preferences (e.g. herding) and (partly) irrational behavior as well as market rigidities for initializing bubbles is explored. A survey on this strand of literature is found in Scherbina (2013).

${ }^{8}$ For example, the Federal Reserve Bank of Dallas employs a real-time monitoring test to detect house price bubbles in its International House Price Database, Grossman et al. (2013)).
} 
be adapted to detect periodically collapsing bubbles (the central criticism of Evans (1991)) by running ADF tests based on forward recursive regressions. More recently, PSY13 generalized this approach by allowing for rolling windows of flexible size for estimation. Both these tests can also be used to date the emergence and collapse of asset price bubbles in real-time 9

The starting point for the tests of PWY11 and PSY13 is a variant of the autoregressive specification

$$
z_{t}=\mu_{z}+\delta z_{t-1}+\sum_{j=1}^{J} \phi_{j} \Delta z_{t-j}+v_{t}, \quad t=1, \ldots, \tau, \quad v_{t} \stackrel{i i d}{\sim} N\left(0, \sigma_{v}^{2}\right)
$$

where $z_{t}=\left\{p_{t}, d_{t}\right\}$ or $z_{t}=\left\{p_{t}-d_{t}\right\}$, with $p_{t}=\ln \left(P_{t}\right)$ and $d_{t}=\ln \left(D_{t}\right)$. All tests employed in this paper are right-tailed ADF-type tests of the null hypothesis $H_{0}: \delta=1$ against the alternative $H_{1}: \delta>1$. In specific, forward recursive regressions of (3) are carried out, providing a sequence of $\mathrm{ADF}$-statistics $A D F_{\tau}$ for all margins $\tau_{0}, \tau_{0}+1, \ldots, T$. Hence, the first regression includes $\tau_{0}=\left\lfloor r_{0} T\right\rfloor$ observations, where $r_{0}$ denotes the minimum share of the total sample size $T$ for which (3) is estimated.

As outlined above, a bubble is detected when the price series shows explosive behavior while the fundamental series does not. The bubble emergence and collapse dates $\left(\tau_{e}\right.$ and $\tau_{f}$ ) can then be estimated as the first date $\tau$ for which the ADF statistic for the price series exceeds (falls below) the critical value $c v_{\alpha_{T}}^{\text {adf }}(\tau)$

$$
\hat{\tau}_{e}=\inf _{\tau \geq \tau_{0}}\left\{\tau: A D F_{\tau}>c v_{\alpha_{T}}^{a d f}(\tau)\right\}, \quad \hat{\tau}_{f}=\inf _{\tau \geq \hat{\tau}_{e}}\left\{\tau: A D F_{\tau}<c v_{\alpha_{T}}^{a d f}(\tau)\right\} .
$$

If the dividend series is explosive at all dates $\tau$ with $\hat{\tau}_{e} \leq \tau \leq \hat{\tau}_{f}$, no bubble is indicated. If the dividend series turns explosive at $\tau$ with $\hat{\tau}_{e}<\tau \leq \hat{\tau}_{f}$, the bubble collapse date $\hat{\tau}_{f}$ is reset to $\hat{\tau}_{f}=\tau-1$. This algorithm yields a binary indicator series $B_{\tau}, \tau=\tau_{0}, \tau_{0}+1, \ldots T$ with

$$
B_{\tau}= \begin{cases}1 & \text { if } \hat{\tau}_{e} \leq \tau \leq \hat{\tau}_{f} \\ 0 & \text { else }\end{cases}
$$

Importantly one can test both series individually (i.e. $z_{t}=p_{t}$ or $z_{t}=d_{t}$ ), or the ratio of the two (i.e. $z_{t}=\left\{p_{t}-d_{t}\right\}$ ). The literature considers both but does not discuss the important implications that follow this choice. ${ }^{10}$ While dividends are typically assumed and found to

\footnotetext{
${ }^{9}$ Along the same lines, Homm \& Breitung (2012) propose a number of flexible tests based on structural breaks in the autoregressive parameters or on forecast breakdowns, two of which can also be employed for real-time monitoring and date-stamping. PSY13 show that these do not provide superior signals compared to the PWY11 and PSY13 approaches and are therefore omitted.

${ }^{10}$ While PWY11 and Harvey et al. (2015) test the series individually, Homm \& Breitung (2012), PWY13 and
} 
follow a random walk (with drift), the log price-dividend ratio is assumed to be stationary (see e.g. Campbell \& Shiller (1988) and Cochrane (1992)). Rewriting (2) into the alternative representation proposed by Campbell \& Shiller (1987) given by

$$
P_{t}-\frac{1}{r} D_{t}=\left(\frac{1+r}{r}\right) \sum_{i=1}^{\infty}\left(\frac{1}{1+r}\right)^{i} E_{t}\left[\Delta D_{t+i}\right]+B_{t}
$$

shows that the difference $P_{t}-\frac{1}{r} D_{t}$ is stationary if $D_{t} \sim I(1)$ and $B_{t}=0$. Hence, it is reasonable to expect that the test on the individual series features a larger power against the null hypothesis than the more conservative test on the ratio. On the one hand, this implies that testing the series individually can provide a more timely detection of a bubble phase. On the other hand, this can also imply a more frequent detection of periods with exponential growth due to short-lived spikes. In this case, a false bubble (or if dividends seem to grow exponentially, a collapse (no bubble)) would be signaled. Thus, testing the $\log$ price-to-dividend ratio can provide information on the relative growth rate of the two series. If both grow exponentially, but prices grow at a faster rate than dividends (indicating increasing imbalances), a unit root test on the ratio would indicate a bubble period that is missed by a test on the individual series. Therefore, this paper evaluates both options.

\section{$2.3 \quad$ Real-time detectors}

\subsubsection{Forward recursive sup ADF Test (PWY11)}

First, the original indicator of Phillips et al. (2011) will be applied by estimating (3) either recursively or by rolling windows. The lag order $J$ is determined by the Akaike Information Criterion (AIC) with $J^{\max }=12$. As with PWY11, this paper will set $r_{0}=\{0.1,0.2\}$ for recursive and rolling-windows estimation, respectively. In order to account for over-rejection in the multiple testing setting, the significance level $\alpha_{T}$ needs to approach zero asymptotically for an overall significance level of $5 \%$ to hold. Correspondingly $c v_{\alpha_{T}}^{a d f}(\tau)$ must diverge to infinity. Thus, PWY11 suggest using $c v_{\alpha_{T}}^{\text {adf }}(\tau)=\ln (\ln (\tau)) / 100$, yielding significance levels around $4 \%$. For the rolling PWY11 indicator, the critical value is a constant $c v_{\alpha_{T}}^{\text {adf }}(\tau)=$ $\ln \left(\ln \left(r_{0} T\right)\right) / 100$.

\subsubsection{Generalized sup ADF Test (PSY13)}

Phillips et al. (2013) extend the work of PWY11 by not only allowing the end point (here $\tau_{2}$ ) to move forward for each recursive regression from $\tau_{0}=\left\lfloor r_{0} T\right\rfloor$ to $T$, but by also allowing the start point $\tau_{1}$ for a given $\tau_{2}$ to vary between all values from 0 to $\max \left(\tau_{2}-\tau_{0}, 0\right)$. Thus, Grossman et al. (2013) run tests on the log price-to-dividend ratio. 
the test augments the forward recursive regressions by estimating and testing all possible backward extending windows from the current margin $\tau_{2}$. For a given end point $\tau_{2} \in\left[\tau_{0}, T\right]$ and the varying start point $\tau_{1} \in\left[0, \tau_{2}-\tau_{0}\right]$, the sequence of ADF test statistics is denoted by $\left\{A D F_{\tau_{1}}^{\tau_{2}}\right\}$. Taking the supremum of this sequence then provides the backward sup ADF statistic for the test margin $\tau_{2}$ denoted by $B S A D F_{\tau_{2}}=\sup _{\tau_{1} \in\left[0, \tau_{2}-\tau_{0}\right]}\left\{A D F_{\tau_{1}}^{\tau_{2}}\right\}$.

Similar to PWY11, emergence and collapse dates are then defined as the first date for which the BSADF statistic exceeds (falls below) the respective right-tailed critical value ${ }^{11}$

$$
\begin{aligned}
& \hat{\tau}_{e}=\inf _{\tau 2 \in\left[\tau_{0}, T\right]}\left\{\tau_{2}: B S A D F_{\tau_{2}}>c v_{\alpha_{T}}^{b s a d f}\left(\tau_{2}\right)\right\}, \\
& \hat{r}_{f}=\inf _{\tau 2 \in\left[\hat{\tau}_{e}, T\right]}\left\{\tau_{2}: B S A D F_{\tau_{2}}<c v_{\alpha_{T}}^{b s a d f}\left(\tau_{2}\right)\right\} .
\end{aligned}
$$

Based on extensive simulations PSY13 suggest an initial sample size of $r_{0}=0.01+\frac{1.8}{\sqrt{T}}$. Following a suggestion of PSY13, the lag order in (3) is fixed and set to $J=1$.

\subsubsection{HP-Filter}

Prior to the seminal contribution of PWY11, the literature defined asset price bubbles predominantly by evaluating the deviation of the real price series from its one-sided HP-filtered trend. This builds on the assumption that the asset's fundamentals follow a slow-moving trend. As such, this indicator can potentially capture periods in which large deviations of prices from their past history occur, regardless of the speed of this accumulation process. Hence, this paper also reevaluates the findings of AWG10, and defines a bubble if the real asset price deviates from its trend beyond a threshold $\kappa_{h p}$, with $\kappa_{h p}=10 \%$ for equity and $\kappa_{h p}=7.5 \%$ for house prices. The smoothing parameter for estimating the trend component of AWG10 is adjusted to monthly frequency and is hence set to $\lambda=100,000 * 3^{4}$. In addition to the recursive method of AWG10, rolling (window size $\omega=96$ ) estimation is also applied. Past estimates of the trend component are not updated as new observations arrive. Dating bubbles using the HP-Filter can be criticized along several dimensions. First, setting the threshold and the smoothing parameter is highly arbitrary. Second, the method can tend to generate more booms in the later part of the sample as the trend estimates becomes less sensitive to new information under recursive estimation. Furthermore, for prolonged or strong bubble periods, the trend component will also capture part of the excessive development, thus underestimating the bubble. Nonetheless, the HP-filter might provide more stable estimates than unit-root tests as it is unlikely to react to short-lived spikes in prices or dividends.

${ }^{11}$ Critical values where simulated using the MATLAB code provided by Shu-Ping Shi on her website (https: //sites.google.com/site/shupingshi/home/research), adapted for the respective sample size $T$ and the parameter $r_{0}$. 


\subsection{Combination of indicators}

PSY13 investigate the finite sample properties of the PSY11 and the PSY13 indicator. The latter is specifically proposed to cope with multiple collapsing bubbles. As PSY13 show, the gains of the PSY13 against the PWY11 indicator are substantial, especially when the first bubble is longer in duration than the second bubble. In these cases the PWY indicator generally only detects the first bubble. However, as PSY13 show, the superiority of their indicator comes with a cost. If there is only one bubble, and if this bubble occurs early in the sample, the PWY11 indicator more often signals the correct number of bubbles. Overall, the PSY13 indicator in this case often detects more than one bubble and may thus provide wrong signals to forecasters.

Against these limitations of the individual indicators, a promising approach to summarize the information content on bubble emergence and collapse is to use combinations. Doing this, one can potentially make use of the different strengths of all indicators regarding the timing and lengths of potential bubbles. Specifically, this paper employs the union set of all individual indicators along different thresholds. A similar approach is proposed by Harvey et al. (2015) for two ex post tests by PWY11 and Homm \& Breitung (2012). In detail, a bubble is detected if any of the two indicators rejects the null hypothesis of a unit root. To assure that the asymptotic size of this union of rejections decision rule is equal to the nominal size, the critical values of each test are rescaled. The necessary rescaling constants are obtained by simulations.

This paper, however, relies on a much larger set of indicators that all potentially entail different valuable information on the development of asset price bubbles in real-time. Hence, an adjustment of critical values by simulations of the limiting distributions of all test statistics is not practical. A possible sidestep around this problem is to explore the detected bubble periods resulting from the union set combinations along different thresholds. Thus, the combined indicator $B_{\tau, \kappa}^{C o m b}$ will signal a bubble in period $\tau$ if at least $\kappa$ individual indicators detect an asset price bubble, i.e.

$$
B_{\tau, \kappa}^{C o m b}= \begin{cases}1 & \text { if } \sum_{\bullet \in \mathfrak{B}} B_{\tau}^{\bullet} \geq \kappa \\ 0 & \text { else }\end{cases}
$$

with $\mathfrak{B}=\left\{P W Y 11_{s}^{i}, P W Y 11_{s}^{r}, P W Y 11_{l}^{i}, P W Y 11_{l}^{r}, P S Y 13^{i}, P S Y 13^{r}, H P_{\text {rec }}, H P_{\text {rol }}\right\}$, $i$ : individual series, $r$ : price-dividend ratio and $\kappa=1, \ldots, K$ with $K \leq|\mathfrak{B}|$. The smaller $\kappa$, the more bubble episodes will be detected, implying an "overdetection". The larger $\kappa$, the more indicators need to signal a bubble, eventually implying an "underdetection". A priori, the choice of the optimal threshold $\kappa$ is not clear. 


\section{Bubble simulations}

Before evaluating the predictive content of these indicators, it is of interest to study their finite sample properties in a controlled experiment. In general, this paper follows PSY13 but it adds to their work along two dimensions. First, not only a price, but also a dividend series is simulated in order to study the effect of testing either both series individually or their log-ratio. Second, this paper not only studies how often the correct number of bubbles is detected, but also assesses the frequency of detecting the true bubble and the delay for signaling its emergence and collapse.

\subsection{Bubbles as mildly explosive processes}

PSY13 study the finite sample power properties of their and the recursive PWY11 indicator against mildly explosive bubble alternatives that are capable of generating a fixed number of bubbles over a specified sample length. For the single bubble case, this process takes the form

$$
\begin{aligned}
z_{t}= & z_{t-1} I\left\{t<\tau_{e}\right\}+\delta_{T} z_{t-1} I\left\{\tau_{e} \leq t \leq \tau_{f}\right\} \\
& +\left(\sum_{k=\tau_{f}+1}^{t} \varepsilon_{k}+z_{\tau_{f}}^{*}\right) I\left\{t>\tau_{f}\right\}+\varepsilon_{t} I\left\{t \leq \tau_{f}\right\},
\end{aligned}
$$

where $\delta_{T}=1+c T^{-\alpha}$ with $c>0$ and $\alpha \in(0,1), \varepsilon_{t} \sim\left(0, \sigma^{2}\right)$ and $z_{\tau_{f}}^{*}=z_{\tau_{e}}+z^{*}$ with $z^{*}=O_{p}(1)$. Until bubble emergence, the process is thus characterized by a random walk. During the bubble period, the process is (mildly) explosive with an expansion rate $\delta_{T}>1$. After the collapse, the process returns to the pre-bubble value plus a small perturbation. As PSY13 emphasize, it is crucial for bubble tests to be able to "restart" after an initial bubble was detected and collapsed. Therefore, a simulation is run that features two explosive and collapsing processes. This two-bubble scenario is described accordingly to the single bubble case with two mildly explosive bubble periods (characterized by the same growth rate $d_{T}$ ) and random walk processes before, in between and after the respective bubble periods

$$
\begin{aligned}
z_{t}= & z_{t-1} I\left\{t \in N_{0}\right\}+\delta_{T} z_{t-1} I\{t \in B 1 \cup B 2\}+\left(\sum_{k=\tau_{1} f+1}^{t} \varepsilon_{k}+z_{\tau_{1} f}^{*}\right) I\left\{t \in N_{1}\right\} \\
& +\left(\sum_{l=\tau_{2} f+1}^{t} \varepsilon_{l}+z_{\tau_{2} f}^{*}\right) I\left\{t \in N_{2}\right\}+\varepsilon_{t} I\left\{t \in N_{0} \cup B_{1} \cup B_{2}\right\}
\end{aligned}
$$

where $N_{0}=\left[1, \tau_{1 e}\right), B_{1}=\left[\tau_{1 e}, \tau_{1 f}\right], N_{1}=\left(\tau_{1 f}, \tau_{2 e}\right), B_{2}=\left[\tau_{2 e}, \tau_{2 f}\right], N_{2}=\left(\tau_{2 f}, T\right]$. In contrast to PSY13, this paper further generates a sequence of dividends that are assumed to follow 
a random walk with drift and drift parameter $\mu=0.38$ that matches the sample estimate for the S\&P500 dividend series. The parameters in (7) and (8) take the values $c=1$ and $\alpha=0.6$ as in PSY13. The processes are initialized with $z_{0}=100$, and are restricted to remain positive throughout. The variance of the disturbance $\varepsilon_{t}$ is matched to the sample standard deviation of the S\&P500 prices series, $\sigma=8.94$. The finite sample properties are evaluated over 5,000 simulations.

\subsection{Single bubble process}

Table 1 shows the average number of bubbles detected in the sample for different sample

lengths $T$, emergence dates $\tau_{e}$ and bubble durations $\tau_{d}$. Overall, most individual indicators signal a bubble more than once on average. With the exception of the HP-filter indicators, the number of detected bubbles increases with bubble duration as expected. This can be driven by two effects. On the one hand, detection becomes more likely the longer a bubble persists. On the other hand, it may also be the case that unit root tests signal bubble emergence, collapse and re-emergence while the bubble continues to run. These on-off signals pose a problem for policy makers. Table 4 discussed below provides some insight into this issue.

Turning to the individual indicators, it is found that the HP-filters signal too many bubbles in the sample (between 2.19-3.91 bubbles on average). This problem is largely independent of the bubble start but worsens the longer the sample and could be addressed by increasing the threshold $\kappa_{h p}$. Similarly, the rolling PWY11 indicator detects too many bubbles on average, but the longer the sample, the fewer bubbles are signaled as the critical value increases with $T$. The PSY13 and recursive PWY11 indicators clearly suffer least from overdetection. In contrast to the study of PSY13, however, the PSY13 indicator generally detects fewer bubbles than the recursive PWY11 indicator. Overall, the PSY13 and recursive PWY11 indicator seem to provide the best signals with only little overdetection. A further key finding of this study is that the choice of the tested series is crucial for detection. As outlined in Section 2.2, testing the individual series has a larger power against the null hypothesis and thus more bubble periods are likely to be observed. As Table 1 shows, this is indeed the case as the tests on log-ratios always detect fewer bubbles than the test on the individual series. Finally, bubble location does not appear to have a large impact on the number of bubbles detected.

From the eight individual indicators, combination indicators are constructed along different threshold levels. The bottom panel of Table 1 shows that cut-off levels of $\kappa=\{4,5,6\}$ provide reasonable results of around one bubble on average. These indicators also seem less affected by bubble location and the sample length compared to individual indicators. However, bubble duration still plays a key role in how many bubbles are detected.

PSY13 analyze the finite sample properties of the indicators along frequency tables 
on the number of bubbles detected. This paper limits the analysis to two simulations with different bubble emergence dates.12 Table 2 shows how often each indicator detects zero, one, two or more bubbles in the sample. Generally, the results displayed mirror Table 1 with the HP-filters and the rolling PWY11 indicator finding too many bubbles, and the recursive PWY11 and the PSY13 indicators detecting the true number of one bubble more frequently. However, the impact of bubble location becomes apparent. As in the study of PSY13, the recursive PWY11 indicator on individual series most frequently detects the true number of bubbles when the bubble occurs early in the sample (in $47.2 \%$ of simulations). In this case, the PSY13 indicator does not detect any bubble in $31.8 \%$ of simulations. When the bubble starts later, however, the PSY13 indicator seems to perform best with a detection accuracy of $52 \%$. Additionally, over- and underdetection seem reasonably balanced. As seen from the lower panel, the combination approach requiring four individual indicators to signal a bubble detects the correct number of bubbles in over $50 \%$ of the cases regardless of the emergence date. Therefore, a combination indicator can provide useful insurance against bubble location.

While the nominal size of the tests is fixed at the $5 \%$ level with the obvious exception of the HP-filter, Table 1 suggests that the rolling PWY11 indicator especially suffers from overdetection. Inspecting averages does not reveal, however, how often the true bubble is detected and how often false alarms are issued. This is assessed by Table 3 . Unsurprisingly, the HP-filter and the rolling PWY11 test on the individual series detect the true bubble most often (in $74 \%$ to $94 \%$ of the cases). As discussed above, this comes, however, at the expense of frequent false signals. For early bubbles, the recursive PWY11 indicator performs better than the PSY13 indicator. However, if the bubble occurs later in the sample or runs longer, the PSY13 indicator performs reasonably well with bubble detection of at least $65 \%$. Again, the combination indicator that requires at least four bubble signals improves bubble detection compared to the PSY13 and the recursive PWY11 while hedging against overdetection as shown in Table 1. Nonetheless, if the bubble runs for less than 20 periods, the chance of missing it is as large as $29 \%$. It may be questionable, however, if short-run bubbles of less than two years (if applied to monthly data) have large macroeconomic impacts.

An obvious concern for policy makers is how stable a bubble signal is during a bubble's run. If an indicator signals a collapse preemptively, an expansive monetary response might end up driving asset prices even higher. Therefore, Table 4 shows the frequency at which an indicator provides more than one signal over the course of an asset price bubble, separated by a signaled collapse. Four issues are apparent: First, the HP-filter provides the most stable signal. Second, the rolling PWY11 filter suffers most from on-off signals. For early and long-

\footnotetext{
${ }^{12}$ Results for detection rates with altering bubble duration and sample lengths are suppressed as they do not provide new insights to the above discussion.
} 
running bubbles, it signals collapses and re-emergences in $55 \%$ of bubbles. Third, instability provides hence a partial explanation for the "overdetection" problem in the unit-root tests indicated in Table 1. Accounting for this issue of overdetection indicates that the recursive PWY11 and PSY13 indicators are roughly of the correct nominal size. Fourth, and lastly, combination indicators do not seem to help against indicator instability.

Finally, Table 5 shows the average delay for each individual indicator until emergence and collapse of the true bubble are signaled. From the left panel, it becomes apparent that the delay until emergence is signaled can be substantial. Applied to monthly data, indicators frequently will miss the bubble over the course of the first year. The earliest signals are generally given by the indicators that signal the most bubbles, which could therefore be due to chance. Importantly, this findings questions whether an early pricking of asset price bubbles, as suggested by proponents of a leaning-against-the-wind policy, is possible. In contrast to this, the right panel shows that most indicators detect the collapse of a bubble almost immediately. Here, it is the recursive PWY11 and the PSY13 indicators applied to individual series that provide the most immediate collapse warnings. Again, bubble location seems to play a role with the PSY13 indicator providing more accurate signals the later a bubble started. Also, the right lower panel shows that combination indicators aggregating the information of four to five individual indicators may improve the detection accuracy with regards to bubble collapse.

All in all, the simulation shows that the recursive PWY11 and PSY13 indicators detect asset price bubbles reasonably accurately without issuing too many false alarms as the rolling PWY11 and the HP-filter. Also, both indicators detect the collapse of asset price bubbles largely on-time. Nonetheless, the HP-filter in particular could provide important additional information, as it suffers from the fewest instabilities and detects asset price bubbles with the shortest delay. Therefore, combination indicators that require at least 4 individual tests to signal a bubble can inform policy makers accurately and hedge against overdetection.

\subsection{Two collapsing bubble processes}

PSY13 show that their proposed indicator is better able to detect multiple collapsing bubbles than the recursive PWY11 indicator. To re-evaluate this and to assess the finite sample power properties of the combination indicator, a simulation is run with sample size $T=200$ and two bubbles of equal length $\tau_{d}=20$ that emerge at $\tau_{1 e}=40$ and $\tau_{2 e}=120$ respectively. The results for the average number of bubbles detected and the frequencies of detecting zero, one, two or more bubbles are displayed in Table 6. Also the frequencies of detecting the first and second bubble are displayed.

As in the single bubble case, the HP-filter and the rolling PWY11 indicators detect too many bubbles on average and signal more than two bubbles in over half of the simulations. 
The PSY13 and the recursive PWY11 indicators, on the other hand, frequently detect less than two bubbles. However, this is more pronounced for the recursive PWY11 than for the PSY13 indicator. This issue becomes clearest when investigating detection rates for the first and the second bubble separately, as given in the last two columns. Here, the finding of PSY13 is confirmed that the PWY11 indicator has difficulties "restarting" after a first bubble and frequently misses the second bubble. In this regard, the PSY13 indicator is clearly superior. Again, testing the individual series provides larger power for detecting bubbles than testing the $\log$ price-to-dividend ratio.

Finally, the bottom panel reveals that combination indicators may offer even larger gains in the multiple bubble scenario compared to the case of a single bubble. Here, the combination indicator with a threshold of $\kappa=3$ most frequently finds two bubbles, yet seems to provide additional false signals. Hence a threshold of $\kappa=3$ or $\kappa=4$ can be suggested.

\section{Macro forecasts using asset price bubble indicators}

The paper will in the following explore the predictive content of bubble periods in stock and house prices in the U.S. The U.S. is chosen as it is the only country for which long series on stock and house prices as well as their fundamentals are readily available on a monthly frequency. Also, financial cycles are considered to be highly pronounced and particularly important for the U.S. (cf. Borio, 2012).

\subsection{Stock and house price bubbles in the U.S.}

The data for stock and housing markets can be obtained from the online data supplement ${ }^{13}$ of Shiller (2005) starting in 1871M01 for stock prices and 1953M01 for house prices. However, the analysis here is restricted to the period from 1975M01 to 2014M07 as the forecasting exercise is carried out for the Great Moderation period starting around 1983M01 14 This paper only looks at broad, aggregate indices since it is bubbles in widely held asset classes that can be expected to to have the largest implications for real economic development through their emergence or unwinding. Thus, the stock price index of interest is the S\&P 500. House prices are obtained from a national index of repeat sales accounting for quality changes published by the U.S. Office of Housing Enterprise Oversight for the period from 1975-1987 and by Fiserv CSV, Inc. since 1987. All individual series are deflated by the U.S. Consumer

\footnotetext{
${ }_{13}$ Available at http://www.econ.yale.edu/ shiller/data.htm.

${ }^{14}$ As AWG10 argue, monetary policy conduct changed significantly with strict inflation targeting during the Volcker regime. Therefore, to explore the feasibility of a leaning-against-a-wind policy, this paper incorporates only sample information from 1983 onwards.
} 
Price Index for All Urban Consumers provided by FRED, Federal Reserve Bank of St. Louis. The choice of the underlying fundamental series for house prices is more controversial than for stock prices. In principal, the "dividend" can be thought of as the rent that an owner saves by living in the house (Himmelberg et al. 2005). However, relying on rent series is problematic as these are generally measured with great error only, or do not account for the intrinsic value of owning a house. Additionally, the causality structure between rental and purchase prices for housing is ambiguous. In case of high market power on the home owners' side, it is possible that rising purchase prices induce rising rents, thus leading to explosive growth in both series during a housing bubble. Eventually, this development is likely not to be sustainable, yet the indicators described above would not signal a bubble. Hence, this paper follows Grossman et al. (2013) and relies on real disposable income per capita as a measure for the fundamental determinant of house prices ${ }^{15}$ The idea behind this is to measure the affordability of housing. Assuming that households devote a constant share of their total income to renting, housing prices can only grow sustainably at the rate of per capita real disposable income. This consideration is in direct spirit of the assessment of financial stability that is the goal of this paper.

From the discussion in the previous section it is clear that there is no universally accepted ex post measure of bubble periods. Hence, it can only be attempted to compare the detected bubble periods to anecdotal evidence. Since 1983M01, these include the run-up in stock prices to the "Black Monday" crash on October 19, 1987; the dot-com bubble that reached its peak in March 2010; and the housing bubble that began deflating in November 2005 (according to real house prices of Shiller (2005)). Note that anecdotal evidence on bubble collapse dates can be provided by investigating turning points of the series, while this is generally not possible for the emergence dates.

Figure 1 shows the detected bubble periods in the S\&P 500. The first finding is that all indicators differ with regard to the detected bubble periods with the HP-filters diverging clearly from the unit-root tests. Yet, some common findings prevail. The pre- "Black Monday" bubble in 1987 is found by three specifications of the PWY11, one specification of the PSY13 indicator and both HP filters. Second, the "dot-com bubble" is detected by all indicators. However, the detection accuracy varies largely with emergence being signaled from as early as 1995M05 to as late as 1996M11 and collapse dates ranging from 1999M08 to 2002M04, about seven months prior and more than two years past the peak in prices. Finally, the rolling PWY11 and the PSY13 applied to log-ratios indicate the financial crisis period from around 2008M10 to 2009M06, all when applied to ratios only though. This period is special as the exponential trend in the price-to-dividend ratio comes from a drastic crash in prices prior to this episode, while dividends decreased only slightly. In that sense, this episodes

${ }^{15}$ The data is obtained from FRED. Series identifier: A229RX0. 
describes a "negative bubble." At the current margin, only the HP-filters indicate a stock market bubble. In sum, the indicators by PWY11 and PSY13 applied to individual series appear to signal the most likely bubble periods. The HP indicators tend to detect too many bubbles to be plausible. Thus, it is reasonable to expect the PWY11 and PSY13 indicators to be most useful for forecasting, if bubble periods indeed matter for real economic outcomes.

Figure 2 shows the combination indicators for the S\&P 500 for $\kappa=1, \ldots, 8$. For $\kappa=1$, several periods that do not feature a prominent increase in the price-to-dividend ratio are classified as bubbles, suggesting overdetection. Increasing the threshold to $\kappa=2$ alleviates this issue, yet the dot-com bubble period extends far beyond the price crash in 2000M3. For $\kappa=\{3,4,5\}$, the pre-crash periods are detected relatively early for the 1987 crash and the 2000 dot-com bubble, and the crisis period in 2008/2009 is identified. Also, the crashes are detected in spot-on. Setting $\kappa=5$ still detects the 1987 and dot-com bubble reasonably early, yet does not indicate the "negative bubble in 2008." Increasing $\kappa$ further deteriorates the emergence detection, especially with $\kappa>5$, as fewer crises are detected and the end of the bubble is frequently signaled before the ultimate peak. It might be suspected that, if at all, indicators with $3 \leq \kappa \leq 5$ are most useful for forecasting.

Figure 3 displays the detected bubble episodes for house prices. When compared to stock price bubbles, the overall picture is less clear. There is wide consensus about the housing price bubble up to around 2006. However, the emergence dates vary from 2000M09 as indicated by the recursive HP-filter to 2003M09 as signaled by the PSY13 applied to the log-ratio. Similarly, the rolling PWY11 indicator signals a collapse at the peak of the price-toincome ratio in 2006M04, while the PSY13 indicators on the individual series find the bubble to last until 2007M02. Beyond that, it is difficult to judge which indicators perform well, as the price-to-income ratio has overall been much more stable with even a small downward trend. It appears though that the PWY13 indicator on the ratio detects far too many bubble episodes while the recursive PWY11 indicator misses the recent bubble that led to the global financial crisis. Overall, the forecast ability of bubble indicators that only detect the recent housing bubble should be limited also due to practical reasons in estimation.

The combination indicators for house prices are shown in Figure 4 . Setting $\kappa \leq 2$ seems to provide too many bubble periods, especially in times when income growth exceeds growth in prices. A similar conclusion can be drawn for $\kappa=3$ with regard to a bubble being detected around 1993. Yet, the number of bubbles is already clearly reduced. With $\kappa=4$ and $\kappa=5$, only the recent housing price bubble starting in late 2001/early 2002 is detected. A priori it is not clear which measure, if any, will be most useful for forecasting which is also due to the practical problems for estimation arising from lack of bubble episodes.

Overall, there are considerable differences between all indicators and their specifications, with few providing continuous bubble periods. Instead, collapse and re-emergence dates 
alternate frequently. ${ }^{16}$ Thus, the lack of continuity will cause forecast models to interpret the termination of a bubble period as a collapse, potentially worsening their predictive ability. A priori it is not clear which indicators predict best, if any. Foremost, it will therefore be of interest to compare the forecast performance of the HP-filters that frequently signal bubbles, to the more cautious unit-root test indicators. Further, it can be expected that stock price indicators are better predictors than house price indicators, simply because model estimation does not suffer from bubble scarcity.

\subsection{Forecasting output and inflation}

The previous section shows that there are promising new measures to detect asset price bubbles in real-time - before their collapse - especially when their information content is aggregated. Hence, it is not entirely clear that an active, leaning-against-the-wind monetary policy is doomed to fail due to the impossibility to identify bubbles. Following the argumentation of Kohn (2006) and AWG10 it is now of interest to evaluate whether these indicators also carry predictive content for central banks' target variables. For the case of the U.S., those target variables are, most importantly, inflation and a measure for real economic activity such as employment or output (Mishkin, 2007). This paper follows AWG10 as closely as possible, investigating the predictive content of bubble indicators for inflation and an output measure, here industrial production. 17

Employing the indicator based on a one-sided, recursive HP filter only, AWG10 do not find any predictive content of stock and house price bubble indicators for predicting inflation, the output gap or output growth 18 Yet, their results potentially suffer from limitations on two dimensions that this paper addresses. First, this paper extends the set of bubble indicators with the ones discussed above. Second, AWG10 do not take into account the realtime dimension of the predictor variables. Most real economic variables, such as industrial production (IP) and employment data, are subject to publication lags and revisions. Stock prices, however, are available in real-time and are not revised ${ }^{19}$

Output (as proxied by IP) and inflation will be predicted by single-equation models

${ }^{16}$ To circumvent this issue, the literature generally suggests bridging bubble periods that are only a few months apart. While this might be feasible employing hindsight in an ex post analysis, it is not possible in a real-time forecasting context.

${ }^{17}$ The Federal Reserve Act of 1977 calls on the Federal Reserve to use its policy instruments "to promote effectively the goals of maximum employment, stable prices, and moderate long-term interest rates" (95th United States Congress, 1977, Sec. 2A). While inflation is the primary target variable for all developed economies' central banks, the mandate of targeting employment is less common and is often addressed through related variables such as output (as in the standard Taylor rule).

${ }^{18}$ In contrast to the finding of Stock \& Watson (2001), AWG10 do find some predictive content of asset prices for output growth when using quarterly growth rates of asset prices as predictors.

${ }^{19}$ The importance of this issue is well documented since the discussion outlined in Croushore \& Stark (2003) and Orphanides (2003). 
including predictors that are most likely included in the information (and target) set of the central bank and are documented as frequently showing significant predictive abilities, as in Stock \& Watson (2001). Hence, the benchmark model against which the marginal predictive content of all bubble indicators is measured includes IP (when inflation is the target), inflation (when output growth is the target), the unemployment rate, the effective funds rate as measure for the policy rate and the term spread between 10-year and 3-months government bond yields. This paper hence differs from AWG10 by adding unemployment and the term spread as key predictors.

Regarding the different real-time availability of the predictors, it is helpful to introduce some notation following Clark \& McCracken (2009). Here, $y_{\tau}(t)$ denotes the vintage $t$ observation of $y$ at time $\tau$, where it must hold that $t \geq \tau$. Hence, for variable $y$ published with a lag of $q$ months, the observations available at time $t$ are $\left\{y_{\tau}(t)\right\}_{\tau=1}^{t-q}$. The full sample then includes observations for the target variable $y$ and the vector of predictors $\left\{\left[y_{\tau}(t), x_{\tau}^{\prime}(t)\right]_{\tau=1}^{t}\right\}_{t=R}^{T}$, where $x_{k}$-specific publication lags and number of revisions are considered (see Table 7). The forecast evaluation is carried out against the final revised values $y_{\tau+h}(T)$ and the evaluation criterion for forecast accuracy is the root mean square prediction error (RMSPE). Direct $h$-step forecasts for $y$ are obtained for each margin $t=R, \ldots, T-h-q$. The evaluation sample is thus of size $P=T-h-q-R+1$. Given this notation, the forecast equation for model $m$ can then be written as

$$
y_{\tau+h}(t)=x_{m, \tau}^{\prime}(t) \beta_{m}+\varepsilon_{m, \tau+h}(t), \quad \varepsilon_{\tau} \stackrel{i i d}{\sim} N\left(0, \sigma_{\varepsilon}^{2}\right)
$$

where the parameter vector $\beta_{m}$ is obtained from recursive least-squares estimation. The benchmark model only includes the "classical" predictors discussed above. This benchmark model is then augmented by all indicators introduced in Section 2, first for stock and house prices separately, and second for a joint indicator taking the value 1 if there is a bubble in either stock or house prices to circumvent the issue of lack of bubble episodes ${ }^{20}$. When an indicator does not signal a single bubble episode in the estimation window $\tau=0, \ldots, t-h-q$ for forecasting margin $t$, the model cannot be estimated. Instead, the benchmark forecast is applied at that margin. That is, the benchmark and the augmented model are identical until the first time that at least one bubble period is detected.

Direct forecasts are applied since an iterative forecasting procedure would require predictions up to $h-1$ for all variables in $x$. While this might be feasible for the benchmark model, forecasting the path of the bubble is not ${ }^{21}$ AWG10 argue that the target horizon

${ }^{20}$ Note that the publication lag of house price bubbles is respected. Also, the models were evaluated using an indicator that adds both stock and house price bubbles. As a joint occurrence of bubbles in both asset classes is rare, this left the results almost unaltered. Finally, the models where evaluated including both bubble indicators separately. Again, the results do not change notably.

${ }^{21}$ In addition, a large literature documents that direct forecasts are more robust to model misspecification (cf. 
of central banks, especially when a leaning-against-the-wind policy should be conducted, includes horizons beyond 2 years. Therefore, monthly forecast are carried out for horizons $h=1,3,6,9,12,18,24,36,48,60$.

Adding to AWG10, this paper also allows for additional lags in the forecasting equation, as it is not obvious that last available data will provide the best forecasts ${ }^{22}$ Specifically, this paper employs a specific-to-general lag length selection following the procedure described by Herwartz (2010). That is, for all lags up to $p=1, \ldots, p_{\max }$, with $p_{\max }=3$, in-sample significant regressors are iteratively added from a minimum set including only a constant until the marginal explanatory content of the variables added is not significantly different from zero. This allows for studying the robustness of the findings to the choice of the benchmark. If benchmark forecasts can be improved more by a flexible lag length selection than by the inclusion of bubble indicators, there is less evidence suggesting a potential for an activist monetary policy.

\subsection{Real-time data description}

As outlined above, the target and predictor variables include monthly observations of industrial production, inflation, the unemployment rate, the effective federal funds rate and the spread between 10-year to 3-month government bond yields. Details on the variables, in particular the publication and revision lags, can be found in Table 7. All real-time data is obtained from the Real-Time Data Set for Macroeconomists of the Federal Reserve Bank of Philadelphia. Real-time data on the unemployment rate are available for monthly observations yet in quarterly vintages, only. As unemployment numbers have always been published in the first week of a month for the sample and revisions are small, a set of monthly vintages is generated. For this, interim monthly vintages are constructed to contain all information from the previous quarterly vintage (that is, assuming no past revisions) and new observations up to publication date from the next quarterly vintage (that is, assuming no future revisions). See Table 8 for an illustration. Similarly, monthly vintages for CPI become available in 1998M11 only. Since 1994Q3, quarterly vintages with monthly observations are published on the Fed Philadelphia website. Those datasets are merged in the same fashion to obtain monthly vintages from the start of the forecasting exercise. Non-revised data is obtained from Federal Reserve Economic Data (FRED) of the Federal Reserve Bank of St. Louis.

All data are transformed to be stationary, if necessary. This implies that industrial production and the consumer price index (CPI) are log-differenced. The stationarity asMarcellino et al., 2006).

${ }^{22}$ Also, a fixed lag length estimation procedure for all variables in $x$ was carried out with $p=0,1, \ldots, p_{\max }$, with $p_{\max }=3$. The results in Section 5 are for $p=0$ only, as this parsimonious model provided the best forecasts for all forecast horizons. For $p>0$, the benchmark forecast and the bubble indicator augmented forecasts deteriorate, leaving the model with $p=0$ to be the toughest benchmark. 
sumption on the remaining variables cannot be rejected consistently by standard tests (ADF and KPSS), hence no transformations are applied 23

Accounting for the change in inflationary regimes around the end of the first term of Paul Volcker as Chairman of the U.S. Fed in 1983, the forecasting exercise will rely only on data starting in 1983M1 with pre-sample data used for variable transformations. Following the period of high inflation, the monetary policy setting is likely to have changed. First evidence that this structural change in monetary policy setting matters also for forecasting was provided by Giacomini \& Rossi (2009), who find that an inflation forecast model building on the Phillips curve suffered from a breakdown from around 1980-1983 when the U.S. Fed drastically increased policy rates to combat high levels of inflation. The initial training sample includes $R=180$ observations, and runs until 1997M12 (the first forecast margin). Hence, the evaluation period includes the dot-com and the housing price bubble, and the crisis period around 2008-2009.

\section{Forecast results}

This section presents the results for the forecasting study outlined above. The predictive content is evaluated separately for stock and house prices, as well as for a joint indicator that signals if there is a bubble in either stock or house prices. Subsequently, the findings are compared for forecasts that consider the real-time availability of the data and forecasts that do not account for this issue.

\subsection{Predictive content for industrial production}

Table 9 displays the predictive accuracy of the benchmark model for IP (first row) and the relative gains and losses of the indicator-augmented models subsequently. Overall, the predictive content differs across the indicators and depends largely on the forecast horizon. For short-run forecasts up to $h=6$, the findings of AWG10 of no predictive content of the recursive HP-indicator are contradicted with small yet significant forecast gains of the bubble augmented model. For larger horizons, however, their findings seem to be supported as the benchmark model performs better. Nonetheless, there are a non-negligible number of models that perform significantly better for both short- and long-run forecasts. Most interestingly, for $h=9, \ldots, 48$, this holds foremost for the PSY13 indicator applied to individual series that finds both the 1987 crash and the dot-com bubble, yet signals some intermediate collapses

${ }^{23}$ The unemployment series does not exhibit a trend over the full sample and all variants of the ADF and KPSS tests accounting for an intercept, suggest stationarity. The real interest rate is found to be trend stationary over the full sample. Due to changing trend estimates depending on the forecast margin, no transformation is applied. The term spread is found to be stationary. 
during the course of the second bubble. The best short-run forecast up to $h=6$ are obtained by the PWY11 indicator with rolling estimation applied to the individual series and by the combination indicator with $\kappa=\{3,4\}]$ that detect the 1987 pre-crash and the dot-com bubble period. Both indicators are extremely accurate in detecting the collapse date, hence the gains potentially stem from the period of lower output growth in the succession of the crisis. Importantly, one can conclude, that these indicators do not only detect plausible bubble periods, but also help significantly in forecasting output growth for all forecast horizons. For indicators that detect either too few or too many bubble periods (such as the combinations with $\kappa \leq 2$ and the HP indicators) the predictive ability deteriorates significantly compared to the benchmark model.

Table 10 displays the results for house price bubble indicators. The recursive PWY11 indicators are suppressed from the results as they do not detect a bubble in the out-of-sample forecast period. Generally, the forecasts slightly deteriorate compared to the benchmark model, especially in the short-run. With the exception of the combination indicator with $\kappa=1$, significant gains can only be obtained in the very long-run ( $h \geq 36)$. As for stock price bubbles, the PWY13 indicator applied to individual series is successful, but only for $h \geq 48$. The poor (short to medium-run) forecast performance of the augmented models can be due to several reasons. First, it is important to emphasize that house prices, in contrast to stock prices, are only available with a lag of two periods and are thus lagging even the real economic variables. Second, the inferior forecast performance can be due to the nature of house price bubbles, which might deflate slower than rapidly crashing stock price bubbles. Also, the rare occurrence of house price bubbles poses difficulties for a proper estimation of the model.

In case stock and house price bubbles share some common features, it might be suitable to combine both asset classes and explore the predictive content of an indicator that signals a bubble as soon as either a stock or house price bubble is detected. These results are displayed in Table 11. Compared to the results in Table 9, forecasts up to $h=18$ are often improved further, especially for the combination indicators with $\kappa=\{3,4,5\}$ and the rolling-window PWY11 indicator applied to individual series. Regarding the longer forecast horizons, the results are ambiguous but indicate a poorer forecast performance for most joint indicators when compared to individual asset price bubble indicators. This is a particular problem for the PSY13 indicator applied to individual series and points to different long-run effects of stock and house price bubbles. Specifically, it is suggested that recessions following house price bubbles are deeper and longer lasting than recessions following stock market crashes, as housing represents an asset class of higher importance to a large share of households.

In order to assess whether the real-time dimension of stock and house price bubble indicators and real variables matter and to compare the findings of this paper to those of 
AWG10, the forecast exercise is carried out for revised data ignoring publication lags. The results are depicted in Table 12 for stock price bubbles and Table 13 for house prices. For both asset classes, the changes from real-time to revised data leave the pattern of forecast gains largely unchanged. Overall, stock price bubbles improve short-run prediction more than house price bubbles, and vice versa. Also the same indicators remain superior. However, the forecast gains for stock prices are now smaller as the overall forecast performance of the benchmark model also improves. For house prices, the opposite holds true. As these are published with a longer lag, their predictive power is now improved relative to the benchmark model. In summary, it is decisive that the real-time dimension is considered in evaluating the forecast performance of asset price bubble indicators.

Finally, the sensitivity of the results with regard to the choice of the benchmark model is assessed. For this, a specific-to-general (SPEC) model selection approach is applied to each forecast model, allowing for a flexible selection of predictors and their lags up to $p_{\max }=$ 3. The results for forecasting IP growth with stock price bubbles are displayed in Table 14. The first thing to notice is that SPEC modeling improves the forecast performance of the benchmark for short horizons up to $h=9$. For these periods, the forecast gains of bubble augmented models decrease, yet the same indicators largely perform better than the benchmark model. However, for $h=3$ and $h=6$, no gains can be achieved. For medium horizons $9 \leq h \leq 18$, the relative forecast gains remain largely in the same range. Beyond that, forecast gains even increase. Similar results hold for SPEC modeling with house price bubbles and the joint indicator of stock and house price bubbles. Hence, it can be concluded, that bubble indicators add predictive content even to models that allow for autoregressive components and additional lags of standard predictors.

\subsection{Predictive content for inflation}

Analogous to the previous section, Table 15 displays the predictive accuracy of stock price bubbles for CPI inflation. In contrast to IP growth, the results here seem to support the finding of AWG10 that bubble indicators do not help to predict inflation in the long-run. However, there are again some indicators that feature significant predictive content in the short and medium term. These include the PSY13 indicator when applied to the price-todividend ratio and the combination indicators when setting a low threshold of $\kappa=1$ or $\kappa=2$. Interestingly, these indicators differ with respect to the identified bubble periods, such that consistent conclusions are difficult to draw from inspecting the RMSPEs only.

Table 16 provides the results for house price indicators. Here, the forecasts are overall very similar to the benchmark model and slightly worse for most indicators for the short and medium prediction horizons. Again, the recursive HP-filter applied by AWG10 does not perform well. The best forecasts are frequently obtained by the rolling HP-filter for $h \geq 12$. 
However, these are most likely due to different parameter estimates only, as the indicator does not detect a bubble in the out-of-sample-forecasting window except for the very last periods. Hence, no additional information is employed for forecasting. If at all, it seems that house price bubbles in matter for medium- and long-run inflation forecasts.

The results for the joint indicator of stock and house price bubbles are displayed in Table 17. Here, the findings are ambiguous. While the forecasts of the rolling HP-filter and the recursive PWY11 indicators improve, especially in the short-run, the combination indicator with $\kappa=2$ now deteriorate compared to the benchmark.

Regarding the implications of considering real-time information when forecasting, the same holds true as for IP forecasts. Evaluating forecasts obtained from revised data of real variables improves the benchmark and cuts down on the predictive gains obtained by including stock price bubble indicators when being cognizant about the real-time dimension. Similarly, the predictive content of house price indicators is exacerbated when revised data is employed. The results described above are also not sensitive to the choice of the benchmark as evaluated by the SPEC modeling approach. This is due to the fact, that the benchmark with SPEC modeling performs worse than the benchmark with fixed lag length for all horizons. Hence there are larger gains possible by including bubble indicators than by a flexible variable and lag length selection without bubble indicators.

\section{Conclusion}

This paper contributes to the discussion on the role of monetary policy in influencing asset price cycles. In particular, there is an ongoing debate on whether central banks should stronger "lean against the wind" of emerging bubbles. For this to be feasible, asset price bubbles need to be detectable in real-time, and these bubble signals should carry predictive content for real variables of interest to central banks.

The paper shows that, following the seminal contribution of Phillips et al. (2011), there is now a promising battery of monitoring tests for detecting asset price bubbles based on recursive unit root tests of prices and their underlying fundamentals. However, the detection and precise dating of asset price bubbles is highly sensitive to the exact specification of the tests. In particular, it is shown in a simulation study that testing prices and dividends separately for explosive roots instead of their log-ratio is clearly superior in detecting bubble processes. Some indicators are also more likely to detect true bubbles than others, which, however, comes at the expense of too many false signals. Hence, this paper proposes aggregating the information contained in the set of individual indicators. The paper shows that these combined indicators generally improve detection accuracy while insuring against the issuance of false signals. Furthermore, this paper highlights that while bubble collapses are detected 
almost immediately, all indicators only signal a bubble emergence with a considerable delay. Therefore, it may be questionable if (monetary) policy makers have the opportunity to react to asset price bubbles as soon as they emerge.

Applied to data for the U.S. stock and housing market, these tests generally detect commonly accepted bubble episodes but often indicate bubbles when there is no strong narrative evidence for such events. Also, the indicators often miss the collapse date of bubbles or signal an end of the bubble prior to the ultimate peak. Again, combination indicators help to aggregate information and reduce uncertainty around the signals.

In a second step, this paper then shows that several of these asset price bubble indicators are also useful for forecasting output growth. Indicators that perform well in predicting output are foremost the Phillips et al. (2013) proposed indicator and the combinations suggested in the present paper. The paper furthermore highlights that the consideration of the real-time dimension of all variables is crucial. As stock prices and dividends are available in real-time, they may particularly add to the information set of central banks. House price bubble indicators that are only available with a lag of two months, suffer from that limitation. Nonetheless, this paper finds that indicators for both asset classes can significantly improve output forecasts obtained from a standard benchmark model. Stock prices are particularly useful in horizons of up to 24 months, while house prices are only useful for even longer horizons. Forecasts for inflation can generally not be improved with the inclusion of asset price bubble indicators.

In sum, these findings suggest that central banks should closely monitor asset price developments for patterns commonly found during bubble episodes. This can then also enrich the information set of central banks and of other practitioners for forecasting. The paper does not answer the question whether central banks can and should pursue an active, leaningagainst-the-wind policy as it remains unclear if monetary policy with its standard tools can effectively contain bubbles without hampering real economic growth in the short-run. 


\section{References}

95th United States Congress (1977). Federal Reserve Reform Act of 1977. An act, Public Law 95-188.

Assenmacher-Wesche, K. \& Gerlach, S. (2010). Monetary Policy and Financial Imbalances: Facts and Fiction. Economic Policy, 25, 437-482.

Borio, C. (2012). The financial cycle and macroeconomics: What have we learnt? BIS Working Papers 395, Bank for International Settlements.

Borio, C. \& Lowe, P. (2004). Securing Sustainable Price Stability: Should Credit Come Back From the Wilderness? BIS Working Papers 157, Bank for International Settlements.

Camerer, C. (1989). Bubbles and Fads in Asset Prices. Journal of Economic Surveys, 3(1), 3-41.

Campbell, J. \& Shiller, R. (1988). The dividend-price ratio and expectations of future dividends and discount factors. Review of Financial Studies, 1(3), 195-228.

Campbell, J. Y. \& Shiller, R. J. (1987). Cointegration and Tests of Present Value Models. Journal of Political Economy, 95(5), pp. 1062-1088.

Clark, T. E. \& McCracken, M. W. (2009). Tests of Equal Predictive Ability With Real-Time Data. Journal of Business Eamp; Economic Statistics, 27(4), 441-454.

Cochrane, J. (1992). Explaining the variance of price-dividend ratios. Review of Financial Studies, $5(2), 243-280$.

Croushore, D. \& Stark, T. (2003). A Real-Time Data Set for Macroeconomists: Does the Data Vintage Matter? The Review of Economics and Statistics, 85(3), 605-617.

Diba, B. T. \& Grossman, H. I. (1988). Explosive Rational Bubbles in Stock Prices? The American Economic Review, 78(3), pp. 520-530.

Draghi, M. (2015). The ECB's recent monetary policy measures: Effectiveness and challenges. Camdessus lecture, IMF, Washington, DC.

Evans, G. W. (1991). Pitfalls in Testing for Explosive Bubbles in Asset Prices. American Economic Review, 81(4), 922-30.

Flood, R. P. \& Hodrick, R. J. (1990). On Testing for Speculative Bubbles. Journal of Economic Perspectives, 4(2), 85-101.

Giacomini, R. \& Rossi, B. (2009). Detecting and Predicting Forecast Breakdowns. Review of Economic Studies, 76(2), 669-705. 
Gürkaynak, R. S. (2008). Econometric Tests of Asset Price Bubbles: Taking Stock. Journal of Economic Surveys, 22(1), 166-186.

Grossman, V., Mack, A., Martínez-García, E., Pavlidis, E., Paya, I., Peel, D., \& Yusupova, A. (2013). Monitoring Housing Markets for Episodes of Exuberance: An Application of the Phillips et al. (2012, 2013) GSADF Test on the Dallas Fed International House Price Database. Working Paper No. 165, Globalization and Monetary Policy Institute, Federal Reserve Bank of Dallas.

Harvey, D. I., Leybourne, S. J., \& Sollis, R. (2015). Recursive Right-Tailed Unit Root Tests for an Explosive Asset Price Bubble. Journal of Financial Econometrics, 13(1), 166-187.

Herwartz, H. (2010). A note on model selection in (time series) regression models - general-to-specific or specific-to-general? Applied Economics Letters, 17(12), 1157-1160.

Himmelberg, C., Mayer, C., \& Sinai, T. (2005). Assessing High House Prices: Bubbles, Fundamentals, and Misperceptions. Working Paper 11643, National Bureau of Economic Research.

Holmstrom, B. \& Tirole, J. (1997). Financial intermediation, loanable funds, and the real sector. The Quarterly Journal of Economics, 112(3), pp. 663-691.

Homm, U. \& Breitung, J. (2012). Testing for Speculative Bubbles in Stock Markets: A Comparison of Alternative Methods. Journal of Financial Econometrics, 10(1), 198-231.

Kohn, D. L. (2006). Monetary Policy and Asset Prices. Speech at "Monetary Policy: A Journey from Theory to Practice," a European Central Bank Colloquium held in honor of Otmar Issing, Frankfurt, Germany.

Marcellino, M., Stock, J. H., \& Watson, M. W. (2006). A comparison of direct and iterated multistep AR methods for forecasting macroeconomic time series. Journal of Econometrics, 135(1-2), 499 -526 .

Mishkin, F. S. (2007). Monetary Policy and the Dual Mandate. Speech at Bridgewater College, Bridgewater, Virginia.

Orphanides, A. (2003). The quest for prosperity without inflation . Journal of Monetary Economics, 50(3), 633 - 663. Swiss National Bank/Study Center Gerzensee Conference on Monetary Policy under Incomplete Information.

Phillips, P. C. B., Shi, S.-P., \& Yu, J. (2013). Testing for Multiple Bubbles: Historical Episodes of Exuberance and Collapse in the SEP 500. Cowles Foundation Discussion Paper 1914.

Phillips, P. C. B., Wu, Y., \& Yu, J. (2011). Explosive Behavior in the 1990's NASDAQ: When Did Exuberance Escalate Asset Values? International Economic Review, 52(1), 201-226.

Scherbina, A. (2013). Asset Price Bubbles: A Selective Survey. Working Paper 13/45, International Monetary Fund. 
Shiller, R. J. (2005). Irrational exuberance. Princeton [u.a.]: Princeton [u.a.] : Princeton Univ. Press, 2. ed. edition. Includes bibliographical references and index.

Stiglitz, J. E. (1990). Symposium on Bubbles. Journal of Economic Perspectives, 4(2), 13-18.

Stock, J. H. \& Watson, M. W. (2001). Forecasting Output and Inflation: The Role of Asset Prices. NBER Working Papers 8180, National Bureau of Economic Research, Inc.

Trichet, J.-C. (2005). Asset price bubbles and monetary policy. Speech at Bridgewater College, Bridgewater, Virginia. 


\section{A Figures}
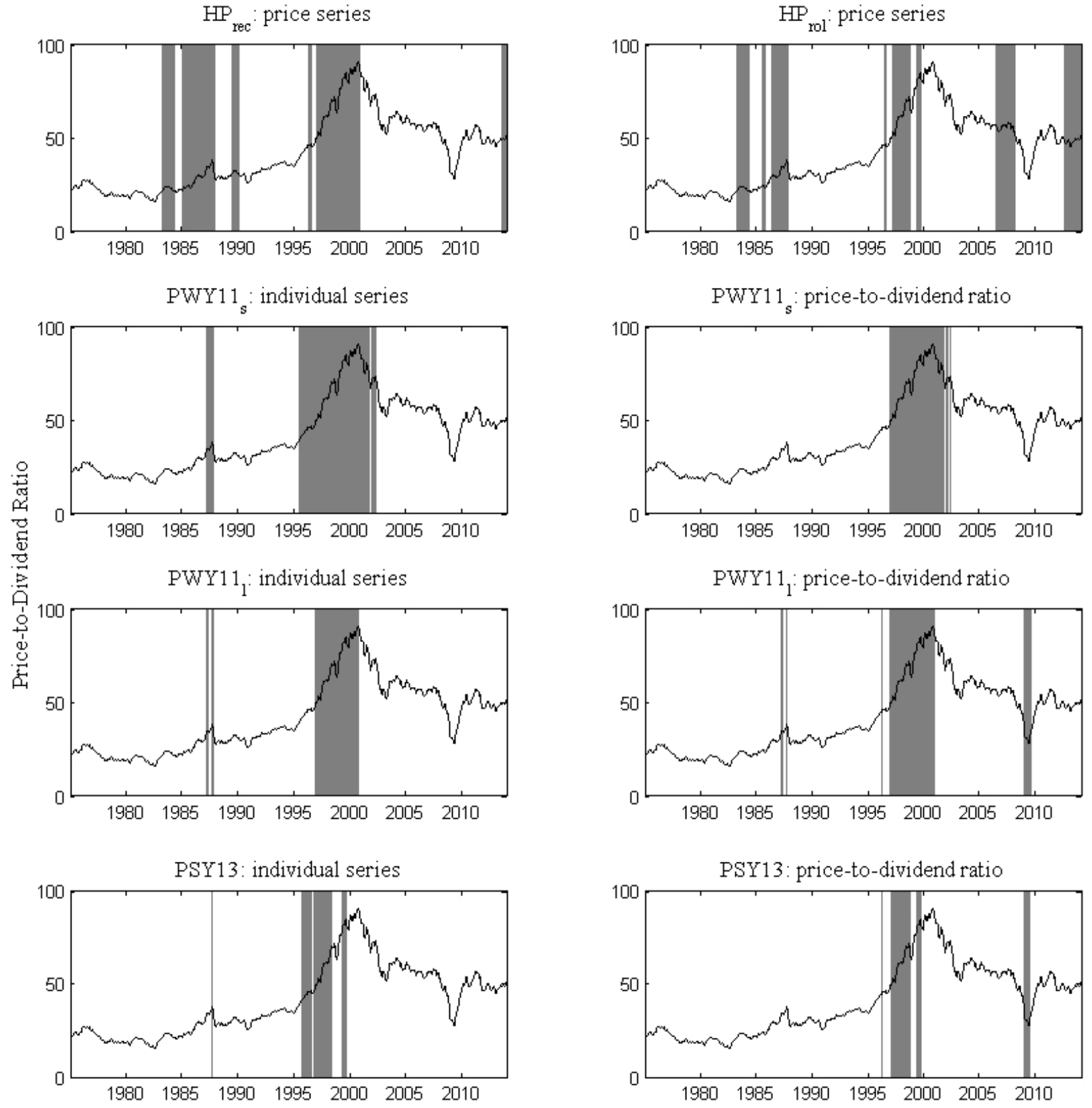

Figure 1: Bubbles in the S\&P 500.

The top panel shows the indicators based on the HP-filter (left: recursive, right: rolling). The bottom three panels show the bubble periods (grey areas) detected by unit root tests. The left bottom panels show the indicators applied to the price and dividend series individually. The right bottom panels shows the indicators applied to the price-to-dividend (PtD) ratio. The solid line plots the PtD ratio as the relevant summary statistic. 

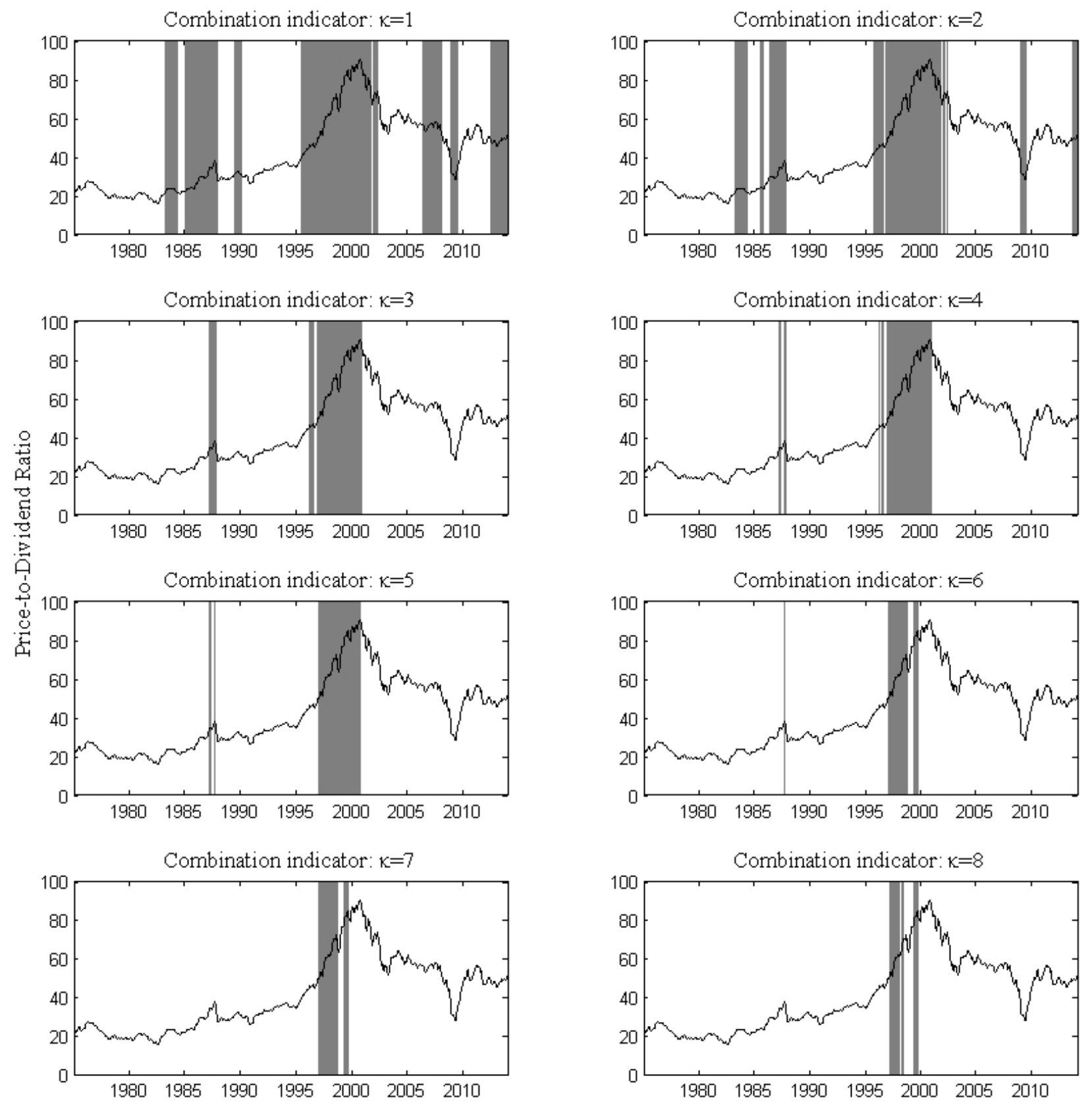

Figure 2: Bubbles in the S\&P 500 as detected by the combination indicators for different threshold levels $\kappa$. The solid line plots the PtD ratio as the relevant summary statistic. 
$\mathrm{HP}_{\text {rec }}$ : price series
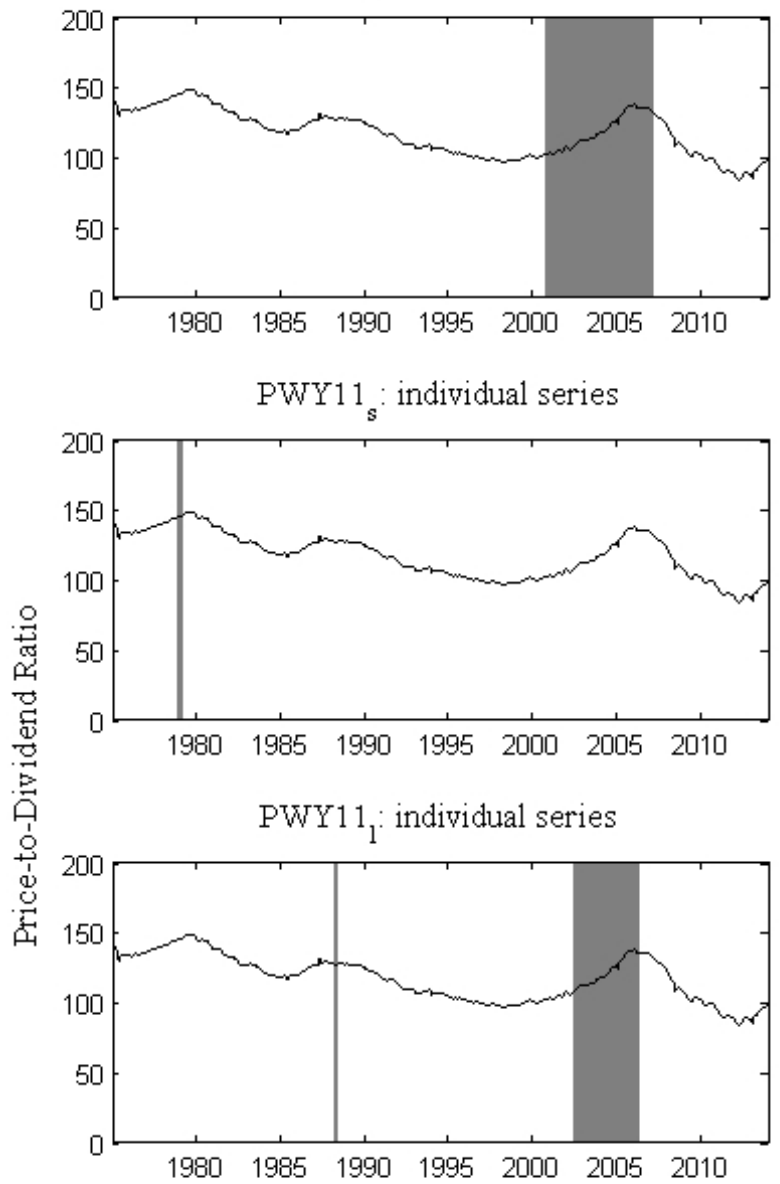

PSY13: individual series

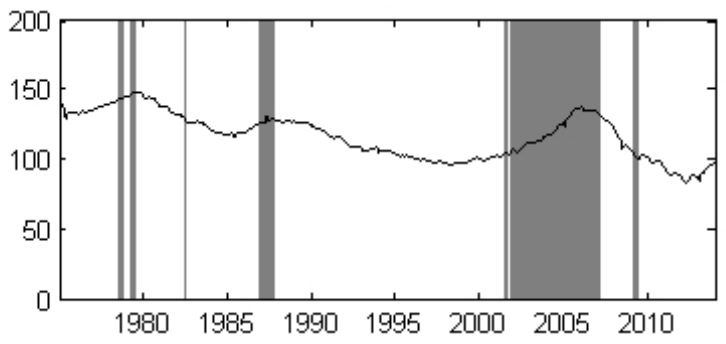

$\mathrm{HP}_{\text {rol }}$ : price series
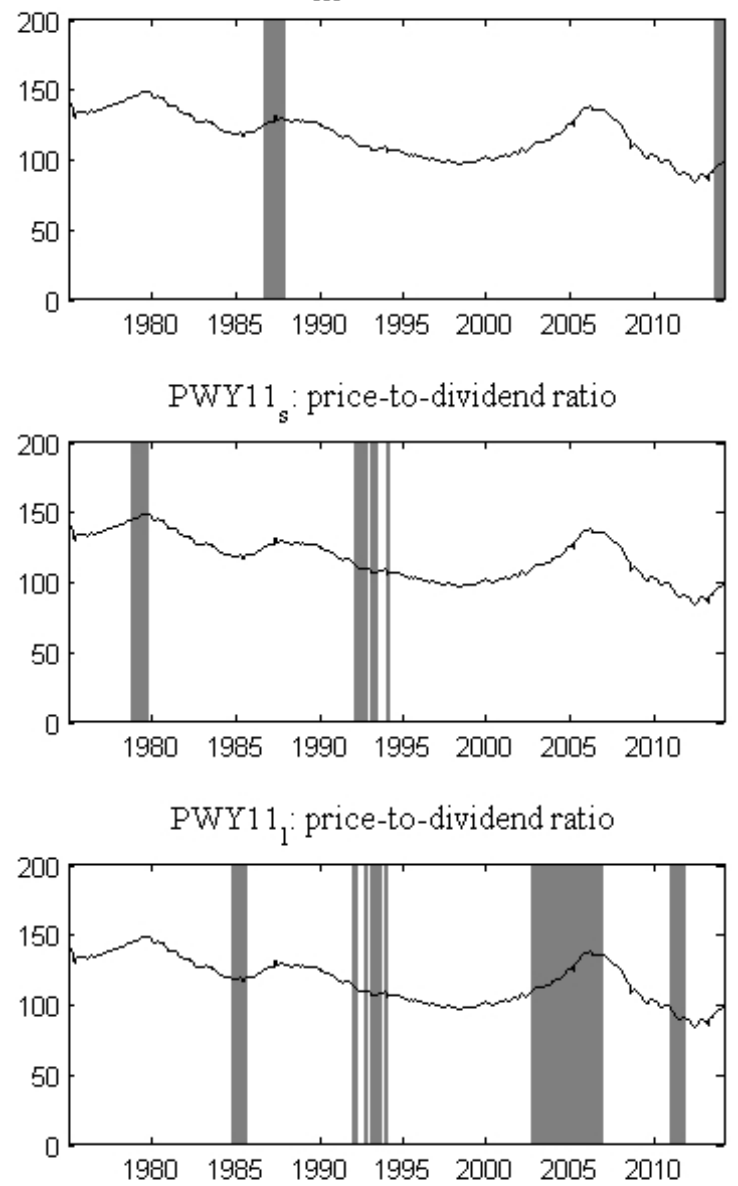

PSY13: price-to-dividend ratio

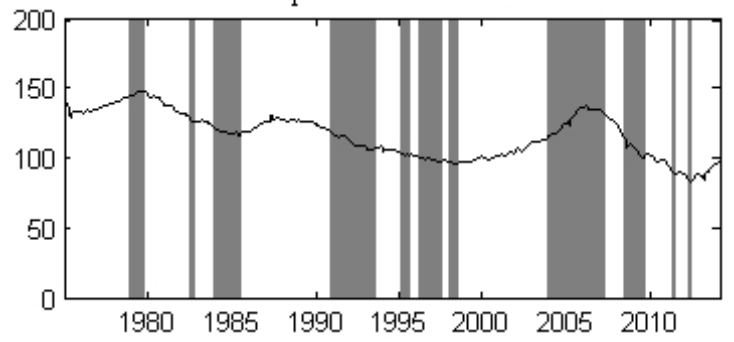

Figure 3: Bubbles in the U.S. housing market. See Figure 1 for further notes. The solid line displays the ratio of average house prices to disposable income per capita. 

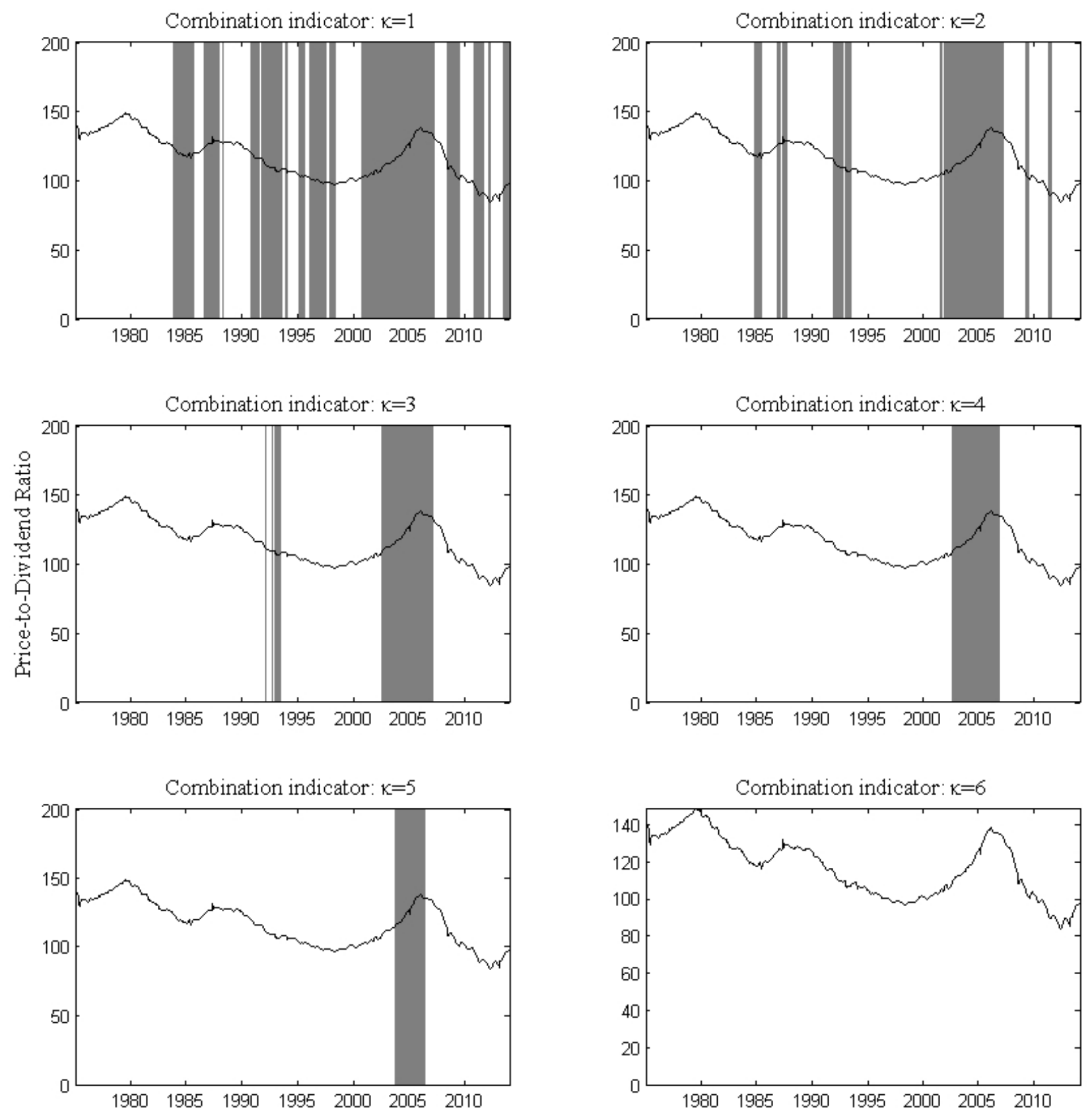

Figure 4: Bubbles in the U.S. housing market as detected by the combination indicators for different threshold levels. The solid line displays the ratio of average house prices to disposable income per capita. 


\section{B Tables}

Table 1: Average number of detected bubbles: One-bubble scenario

\begin{tabular}{|c|c|c|c|c|c|c|}
\hline Indicator & \multicolumn{6}{|c|}{ Average number of detected bubbles } \\
\hline Sample size $T$ & 200 & 200 & 200 & 200 & 300 & 300 \\
\hline Emergence date $\tau_{e}$ & 40 & 40 & 120 & 120 & 60 & 180 \\
\hline Duration $\tau_{d}$ & 20 & 40 & 20 & 40 & 30 & 30 \\
\hline $\mathrm{HP}_{\text {rec }}$ & 2.49 & 2.19 & 2.43 & 2.23 & 3.33 & 3.08 \\
\hline $\mathrm{HP}_{\text {rol }}$ & 2.80 & 2.77 & 2.53 & 2.25 & 3.83 & 3.91 \\
\hline PWY11 ${ }_{\mathrm{s}}$ : individual & 1.20 & 1.56 & 1.26 & 1.65 & 1.16 & 1.19 \\
\hline PWY11 $:$ ratio & 1.08 & 1.53 & 1.17 & 1.53 & 0.93 & 1.01 \\
\hline PWY11 1 : individual & 3.09 & 3.45 & 3.06 & 3.51 & 2.87 & 2.86 \\
\hline PWY11 $:$ ratio & 2.97 & 3.40 & 3.05 & 3.44 & 2.45 & 2.60 \\
\hline PSY13: individual & 1.00 & 1.45 & 1.09 & 1.43 & 1.18 & 1.31 \\
\hline PSY13: ratio & 0.71 & 1.24 & 0.80 & 1.41 & 0.86 & 1.08 \\
\hline Combination: $\kappa$ & 5.20 & 4.26 & 5.16 & 4.34 & 5.56 & 5.44 \\
\hline Combination: $\kappa=2$ & 3.28 & 3.09 & 3.21 & 2.87 & 3.61 & 3.63 \\
\hline Combination: $\kappa=3$ & 1.95 & 2.06 & 2.02 & 2.17 & 1.92 & 2.01 \\
\hline Combination: $\kappa=4$ & 1.16 & 1.64 & 1.27 & 1.70 & 1.24 & 1.37 \\
\hline Combination: $\kappa=5$ & 0.73 & 1.54 & 0.75 & 1.51 & 0.84 & 0.95 \\
\hline Combination: $\kappa=6$ & 0.43 & 1.20 & 0.44 & 1.23 & 0.50 & 0.65 \\
\hline Combination: $\kappa=7$ & 0.21 & 0.76 & 0.18 & 0.73 & 0.29 & 0.32 \\
\hline Combination: $\kappa=8$ & 0.08 & 0.30 & 0.04 & 0.21 & 0.12 & 0.08 \\
\hline
\end{tabular}

The table shows the average number of detected bubbles in the onebubble simulation exercise.

Rows 2-4 indicate the sample size, the emergence date and duration of the bubble.

Rows 5-12 display the results for the individual indicators, rows 13-20 for the combination indicators with different thresholds $\kappa$.

Bold entries indicate the best indicator in each category. 
Table 2: Frequency of detecting zero, one, two or more bubbles: One-bubble scenario

\begin{tabular}{l|cccc|cccc}
\hline \hline & \multicolumn{7}{|c}{ Number of detected bubbles } \\
& Zero & One & Two & More & Zero & One & Two & More \\
\hline Emergence date & \multicolumn{6}{|c|}{$\tau_{e}=40$} & & \multicolumn{4}{c}{$\tau_{e}=120$} \\
\hline HP $_{\text {rec }}$ & 0.021 & 0.221 & 0.319 & 0.439 & 0.004 & 0.249 & 0.331 & 0.417 \\
HP $_{\text {rol }}$ & 0.006 & 0.085 & 0.348 & 0.560 & 0.001 & 0.216 & 0.328 & 0.455 \\
PWY11 : individual & 0.216 & $\mathbf{0 . 4 7 2}$ & 0.231 & 0.080 & 0.259 & 0.403 & 0.211 & 0.126 \\
PWY11 : ratio & 0.342 & 0.372 & 0.192 & 0.093 & 0.338 & 0.354 & 0.180 & 0.129 \\
PWY11 $:$ individual & 0.026 & 0.125 & 0.234 & 0.615 & 0.024 & 0.130 & 0.229 & 0.617 \\
PWY11 $:$ ratio & 0.042 & 0.134 & 0.239 & 0.585 & 0.026 & 0.130 & 0.237 & 0.607 \\
PSY13: individual & 0.318 & 0.443 & 0.175 & 0.065 & 0.229 & $\mathbf{0 . 5 2 0}$ & 0.194 & 0.058 \\
PSY13: ratio & 0.517 & 0.323 & 0.112 & 0.049 & 0.453 & 0.359 & 0.140 & 0.049 \\
\hline Combination: $\kappa=1$ & 0.000 & 0.001 & 0.031 & 0.968 & 0.000 & 0.005 & 0.041 & 0.954 \\
Combination: $\kappa=2$ & 0.002 & 0.098 & 0.234 & 0.666 & 0.001 & 0.118 & 0.245 & 0.636 \\
Combination: $\kappa=3$ & 0.052 & 0.369 & 0.307 & 0.272 & 0.036 & 0.365 & 0.308 & 0.291 \\
Combination: $\kappa=4$ & 0.208 & $\mathbf{0 . 5 1 6}$ & 0.198 & 0.077 & 0.172 & $\mathbf{0 . 5 0 2}$ & 0.235 & 0.091 \\
Combination: $\kappa=5$ & 0.422 & 0.447 & 0.112 & 0.019 & 0.406 & 0.461 & 0.114 & 0.019 \\
Combination: $\kappa=6$ & 0.655 & 0.269 & 0.065 & 0.011 & 0.641 & 0.290 & 0.063 & 0.006 \\
Combination: $\kappa=7$ & 0.819 & 0.150 & 0.029 & 0.002 & 0.846 & 0.131 & 0.021 & 0.002 \\
Combination: $\kappa=8$ & 0.928 & 0.064 & 0.007 & 0.000 & 0.962 & 0.034 & 0.004 & 0.001 \\
\hline \hline
\end{tabular}

The table shows the frequency with which an indicator detects zero, one, two or more bubbles in the one-bubble simulation exercise with sample size $T=200$, duration $\tau_{d}=20$ and either an early (left panel) or a late bubble start (right panel).

Row 2 indicates the emergence date.

Rows 3-10 display the results for the individual indicators, rows 11-18 for the combination indicators with different thresholds $\kappa$.

Bold entries indicate the best indicator in each category. 
Table 3: Frequency of detecting the true bubble: One-bubble scenario

\begin{tabular}{|c|c|c|c|c|c|c|}
\hline Indicator & \multicolumn{6}{|c|}{ Frequency of detecting true bubble } \\
\hline Sample Size $T$ & 200 & 200 & 200 & 200 & 300 & 300 \\
\hline Emergence date $\tau_{e}$ & 40 & 40 & 120 & 120 & 60 & 180 \\
\hline Duration $\tau_{d}$ & 20 & 40 & 20 & 40 & 30 & 30 \\
\hline $\mathrm{HP}_{\text {rec }}$ & 0.83 & 0.91 & 0.78 & 0.83 & 0.85 & 0.82 \\
\hline $\mathbf{H P}_{\text {rol }}$ & 0.83 & 0.91 & 0.80 & 0.84 & 0.85 & 0.85 \\
\hline PWY11 : individual & 0.62 & 0.87 & 0.44 & 0.82 & 0.64 & 0.49 \\
\hline PWY11 $:$ ratio & 0.33 & 0.66 & 0.14 & 0.45 & 0.31 & 0.14 \\
\hline PWY11 1 : individual & 0.74 & 0.92 & 0.78 & 0.94 & 0.80 & 0.87 \\
\hline PWY11 $:$ ratio & 0.53 & 0.88 & 0.64 & 0.91 & 0.50 & 0.67 \\
\hline PSY13: individual & 0.56 & 0.84 & 0.65 & 0.88 & 0.61 & 0.74 \\
\hline PSY13: ratio & 0.26 & 0.59 & 0.32 & 0.68 & 0.29 & 0.43 \\
\hline Combination: $\kappa=1$ & 0.98 & 1.00 & 0.75 & 0.76 & 0.99 & 0.75 \\
\hline Combination: $\kappa=2$ & 0.97 & 1.00 & 0.82 & 0.86 & 0.98 & 0.90 \\
\hline Combination: $\kappa=3$ & 0.83 & 0.99 & 0.84 & 0.96 & 0.85 & 0.90 \\
\hline Combination: $\kappa=4$ & 0.71 & 0.94 & 0.73 & 0.95 & 0.75 & 0.82 \\
\hline Combination: $\kappa=5$ & 0.55 & 0.87 & 0.53 & 0.85 & 0.60 & 0.67 \\
\hline Combination: $\kappa=6$ & 0.33 & 0.71 & 0.33 & 0.70 & 0.37 & 0.46 \\
\hline Combination: $\kappa=7$ & 0.18 & 0.49 & 0.15 & 0.47 & 0.22 & 0.25 \\
\hline Combination: $\kappa=8$ & 0.07 & 0.23 & 0.04 & 0.16 & 0.09 & 0.06 \\
\hline
\end{tabular}

The table shows the frequency with which each indicator detects the true bubble in the one-bubble simulation exercise.

Higher detection frequency generally comes at the cost of more type-I errors of detecting a bubble when no bubble is present.

For further notes, see Table 1 . 
Table 4: Frequency of on-off signals during true bubble: One-bubble scenario

\begin{tabular}{l|cccccc}
\hline \hline Indicator & \multicolumn{7}{|c}{ Frequency of } & on-off signals \\
\hline Sample Size $T$ & 200 & 200 & 200 & 200 & 300 & 300 \\
Emergence date $\tau_{e}$ & 40 & 40 & 120 & 120 & 60 & 180 \\
Duration $\tau_{d}$ & 20 & 40 & 20 & 40 & 30 & 30 \\
\hline HP $_{\text {rec }}$ & 0.03 & 0.07 & 0.01 & 0.01 & 0.06 & 0.01 \\
HP $_{\text {rol }}$ & 0.03 & 0.07 & 0.01 & 0.01 & 0.06 & 0.02 \\
PWY11 $_{\text {s }}$ individual & 0.09 & 0.21 & 0.01 & 0.04 & 0.10 & 0.01 \\
PWY11 $:$ ratio & 0.07 & 0.25 & 0.02 & 0.09 & 0.08 & 0.02 \\
PWY11 $:$ individual & 0.20 & 0.55 & 0.24 & 0.59 & 0.29 & 0.30 \\
PWY11 $:$ ratio & 0.13 & 0.46 & 0.16 & 0.52 & 0.15 & 0.23 \\
PSY13: individual & 0.10 & 0.27 & 0.08 & 0.20 & 0.15 & 0.13 \\
PSY13: ratio & 0.05 & 0.24 & 0.07 & 0.28 & 0.08 & 0.13 \\
\hline Combination: $\kappa=1$ & 0.16 & 0.20 & 0.07 & 0.08 & 0.16 & 0.05 \\
Combination: $\kappa=2$ & 0.14 & 0.18 & 0.05 & 0.06 & 0.14 & 0.06 \\
Combination: $\kappa=3$ & 0.14 & 0.30 & 0.12 & 0.25 & 0.17 & 0.15 \\
Combination: $\kappa=4$ & 0.13 & 0.36 & 0.13 & 0.30 & 0.18 & 0.17 \\
Combination: $\kappa=5$ & 0.11 & 0.45 & 0.09 & 0.39 & 0.17 & 0.15 \\
Combination: $\kappa=6$ & 0.07 & 0.34 & 0.06 & 0.35 & 0.10 & 0.13 \\
Combination: $\kappa=7$ & 0.03 & 0.21 & 0.02 & 0.20 & 0.06 & 0.06 \\
Combination: $\kappa=8$ & 0.01 & 0.06 & 0.00 & 0.04 & 0.02 & 0.01 \\
\hline \hline
\end{tabular}

The table shows the how often an indicator signals a collapse and re-emergence during the course of the true bubble.

For further notes, see Table 1 . 


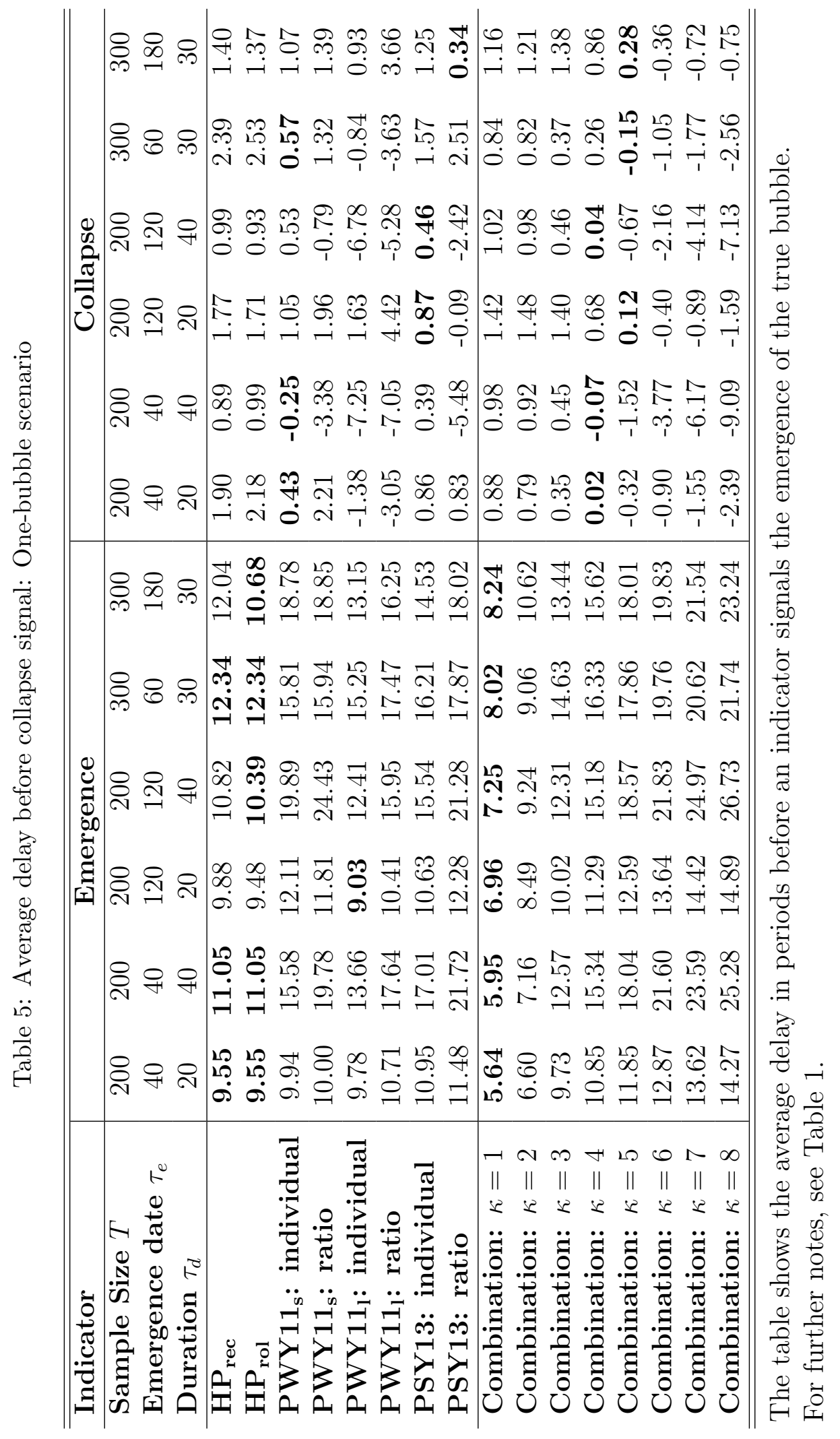




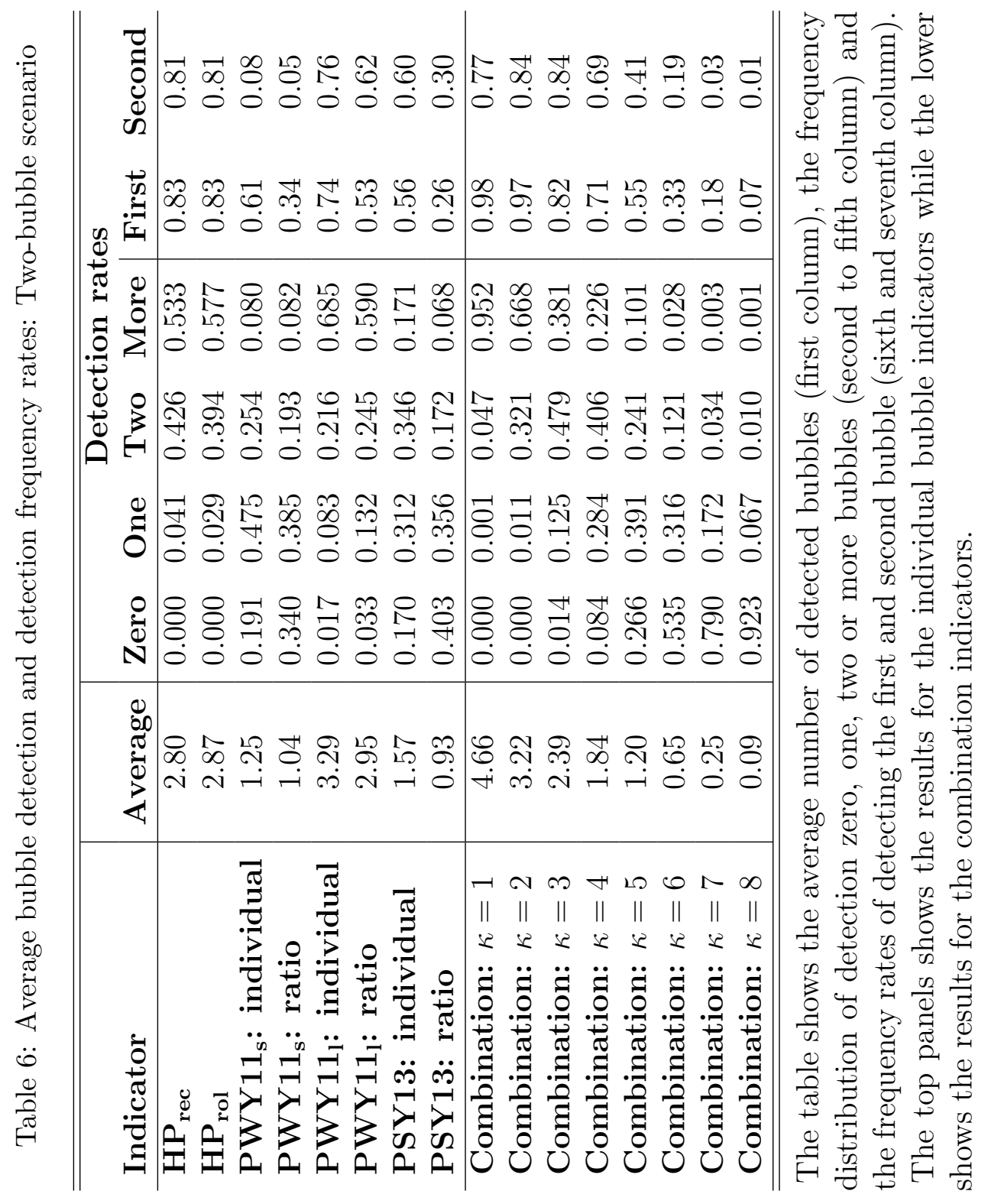




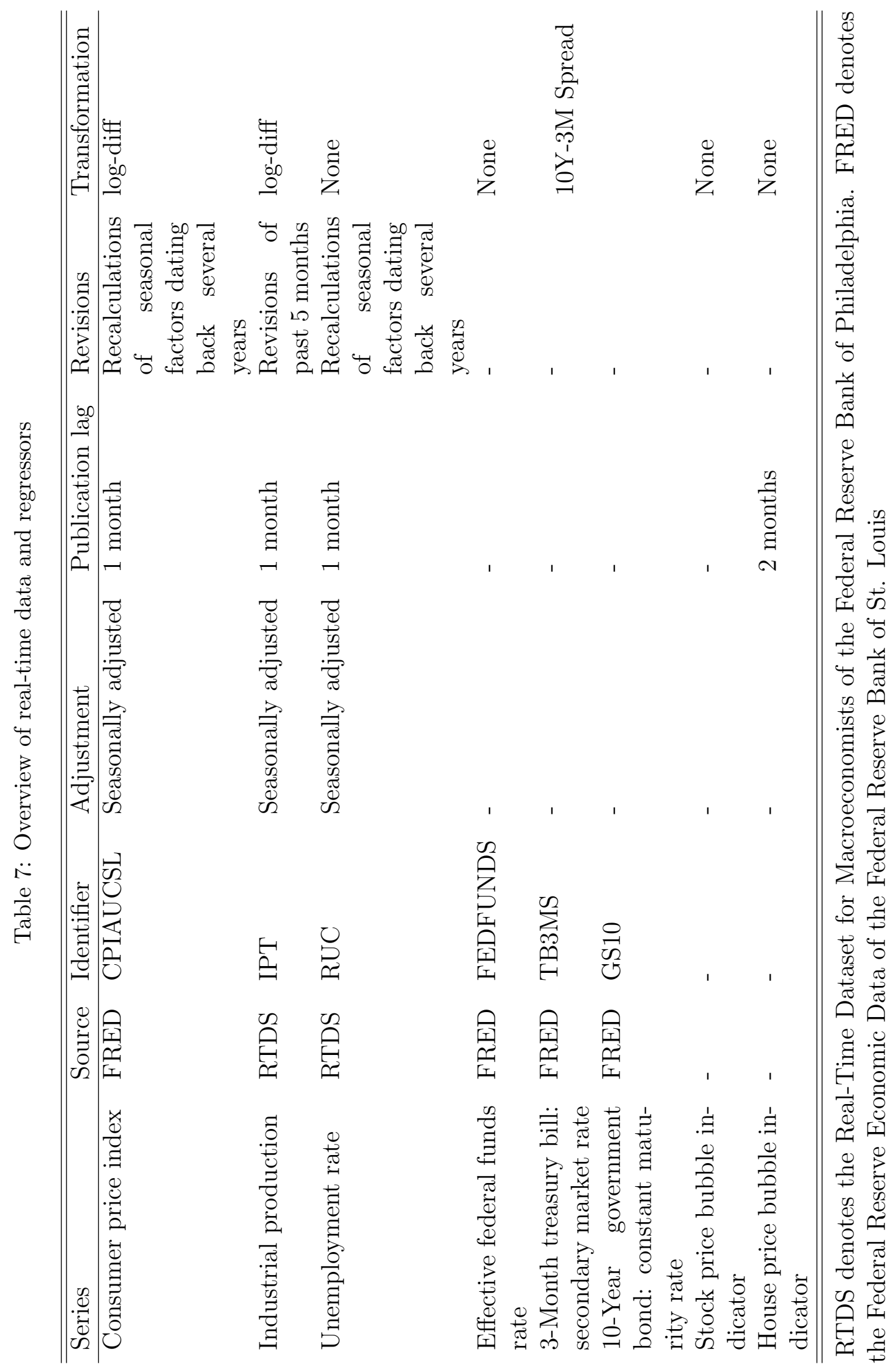


Table 8: Transforming quarterly vintages of unemployment data to monthly vintages

\begin{tabular}{|c|c|c|c|c|c|c|c|c|}
\hline & \multicolumn{8}{|c|}{ Monthly vintages } \\
\hline Date & M1 & $\mathrm{Q} 1=\mathrm{M} 2$ & M3 & M4 & $\mathrm{Q} 2=\mathrm{M} 5$ & M6 & M7 & $\ldots$ \\
\hline M1 & - & $U_{M 1}(Q 1)$ & $U_{M 1}(Q 1)$ & $U_{M 1}(Q 1)$ & $U_{M 1}(Q 2)$ & $U_{M 1}(Q 2)$ & $U_{M 1}(Q 2)$ & $\ldots$ \\
\hline M2 & - & - & $U_{M 2}(Q 2)$ & $U_{M 2}(Q 2)$ & $U_{M 2}(Q 2)$ & $U_{M 2}(Q 2)$ & $U_{M 2}(Q 2)$ & $\ldots$ \\
\hline M3 & - & - & - & $U_{M 3}(Q 2)$ & $U_{M 3}(Q 2)$ & $U_{M 3}(Q 2)$ & $U_{M 3}(Q 2)$ & . . \\
\hline M4 & - & - & - & - & $U_{M 4}(Q 2)$ & $U_{M 4}(Q 2)$ & $U_{M 4}(Q 2)$ & $\ldots$ \\
\hline M5 & - & - & - & - & - & $U_{M 5}(Q 3)$ & $U_{M 5}(Q 3)$ & .. \\
\hline M6 & - & - & - & - & - & - & $U_{M 6}(Q 3)$ & $\ldots$ \\
\hline
\end{tabular}

The table explains how quarterly vintage data of monthly observations of the unemployment rate $U$ are transformed to monthly vintages. The subscript denotes the observation date, the term in bracket denotes the quarterly vintage from which the observation is obtained. The unemployment rate is published in the first week of the following month. 


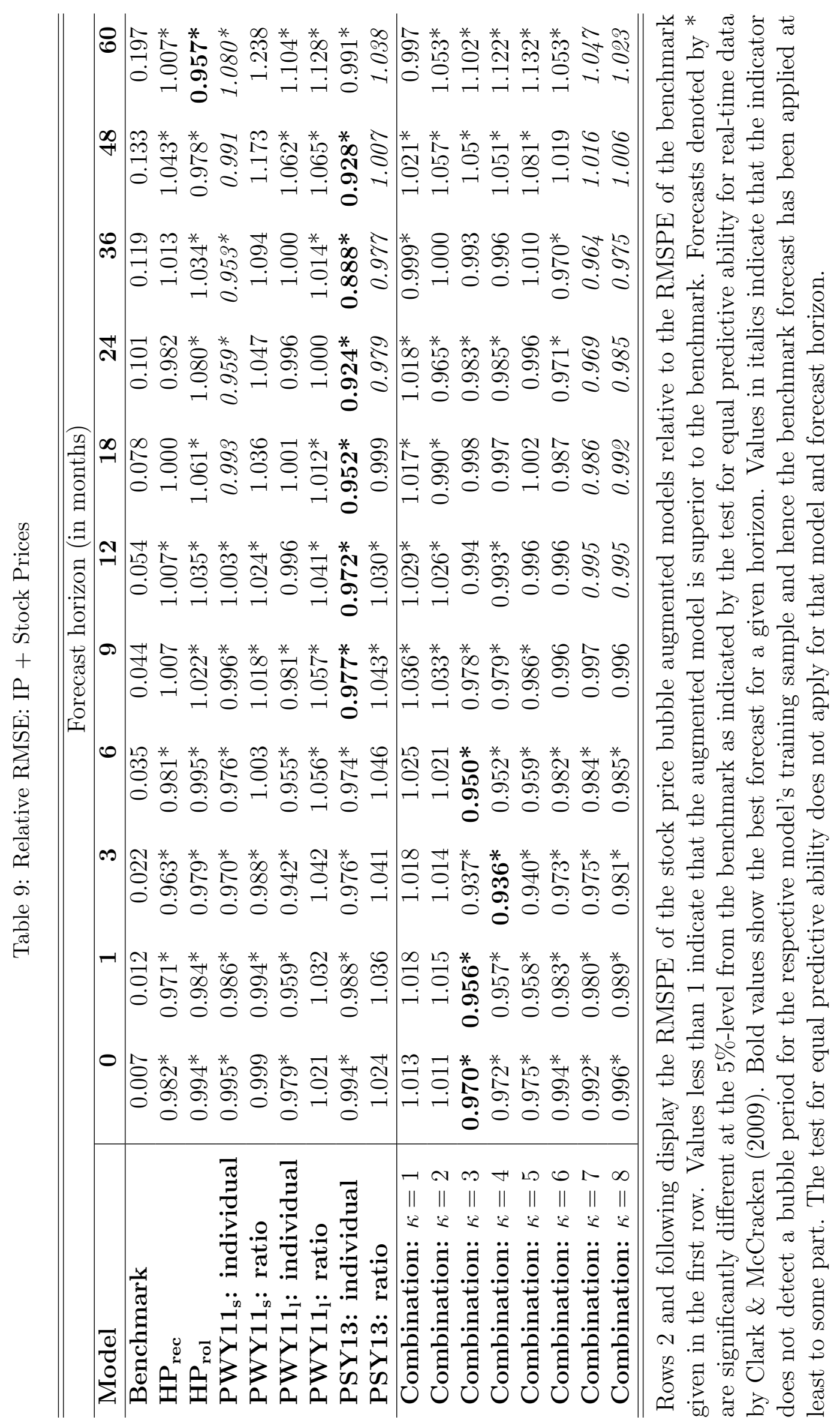




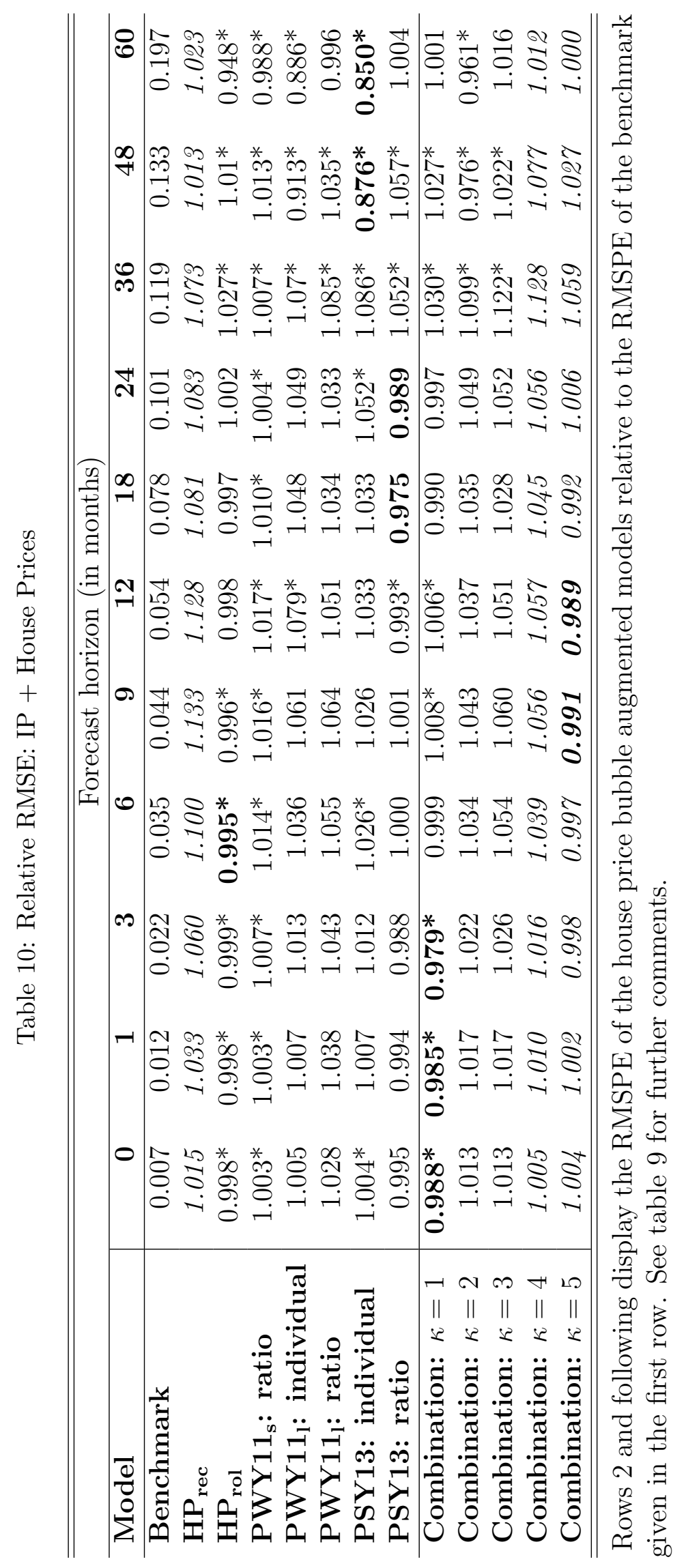




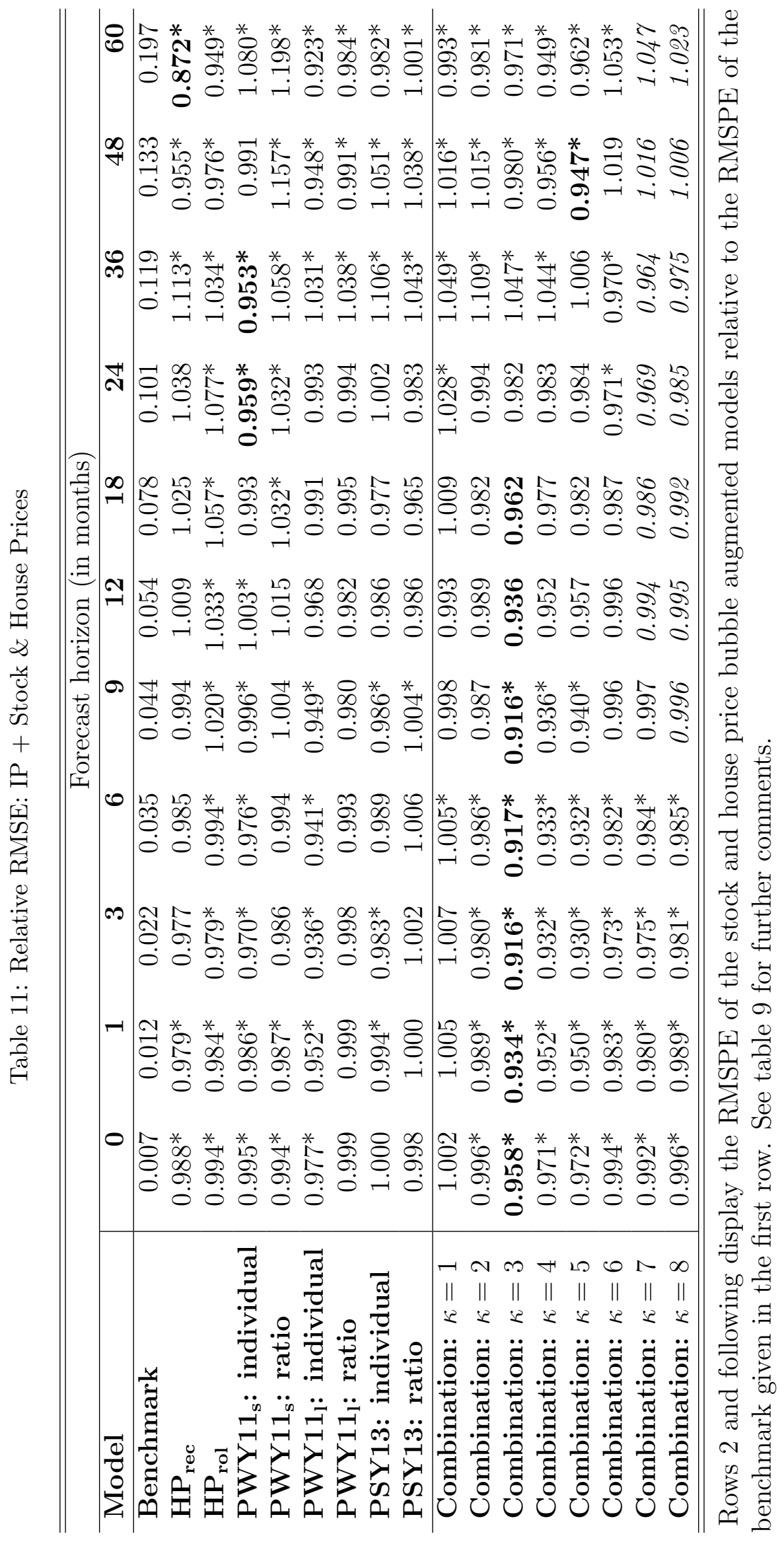




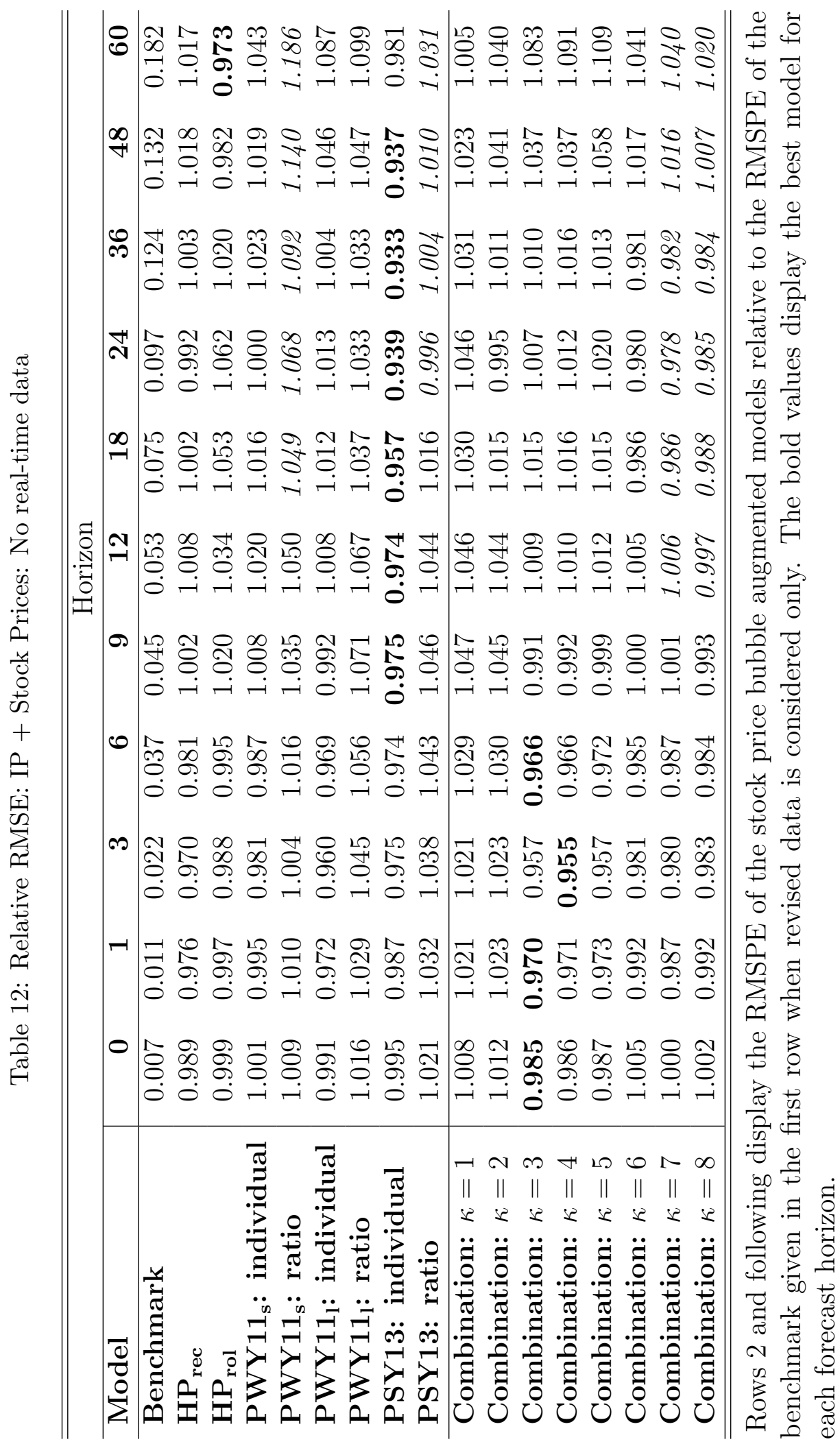




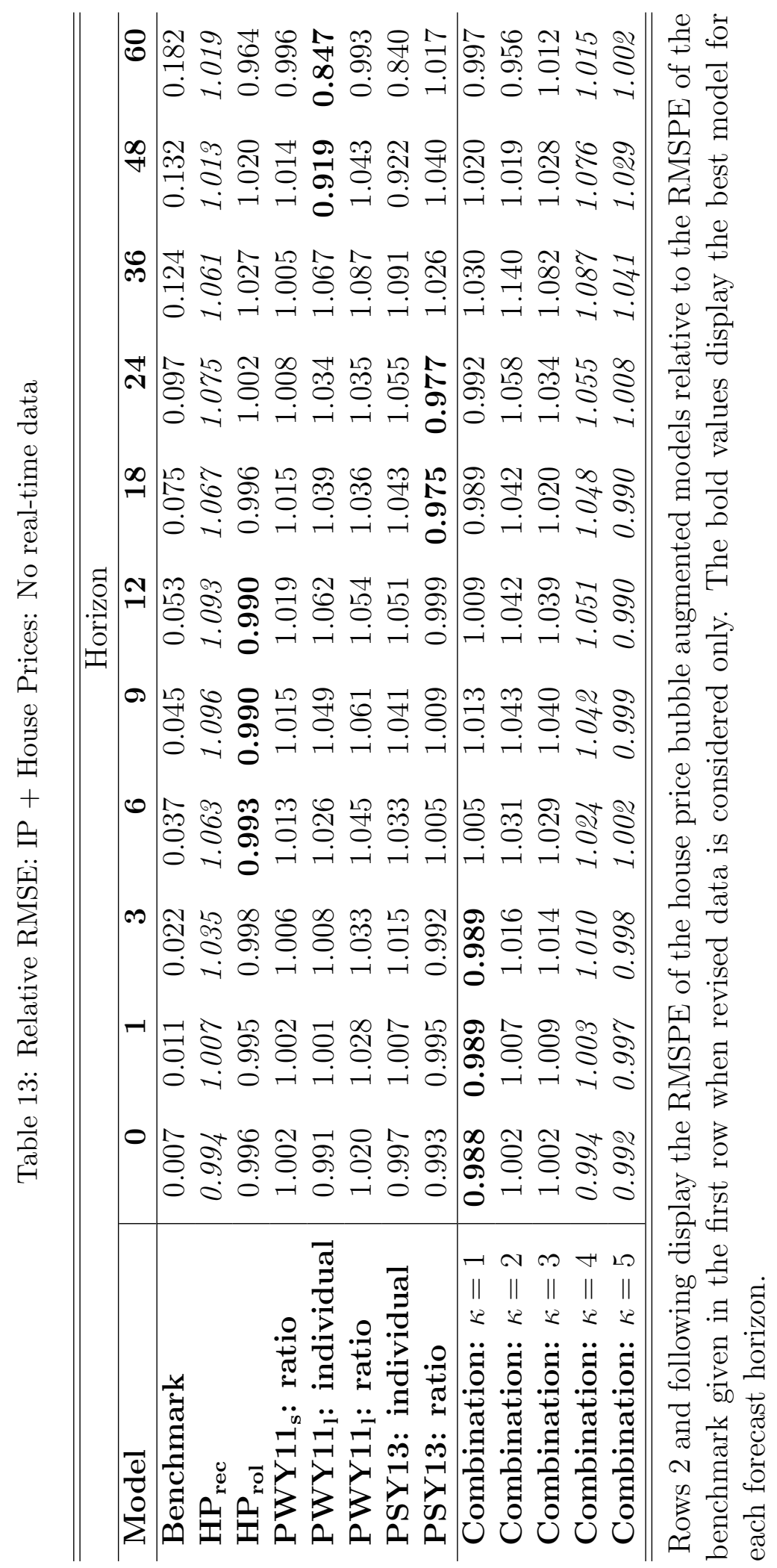




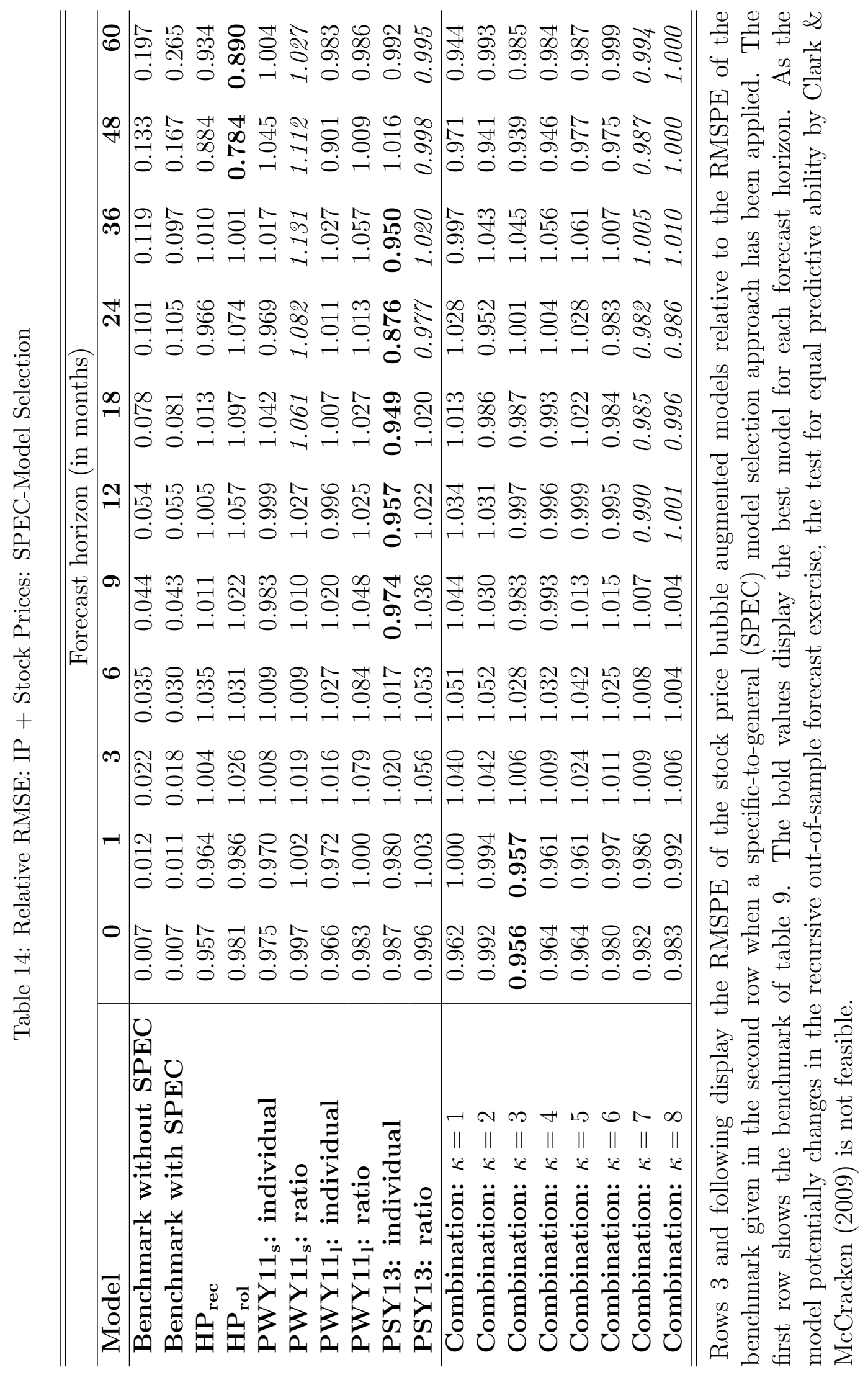




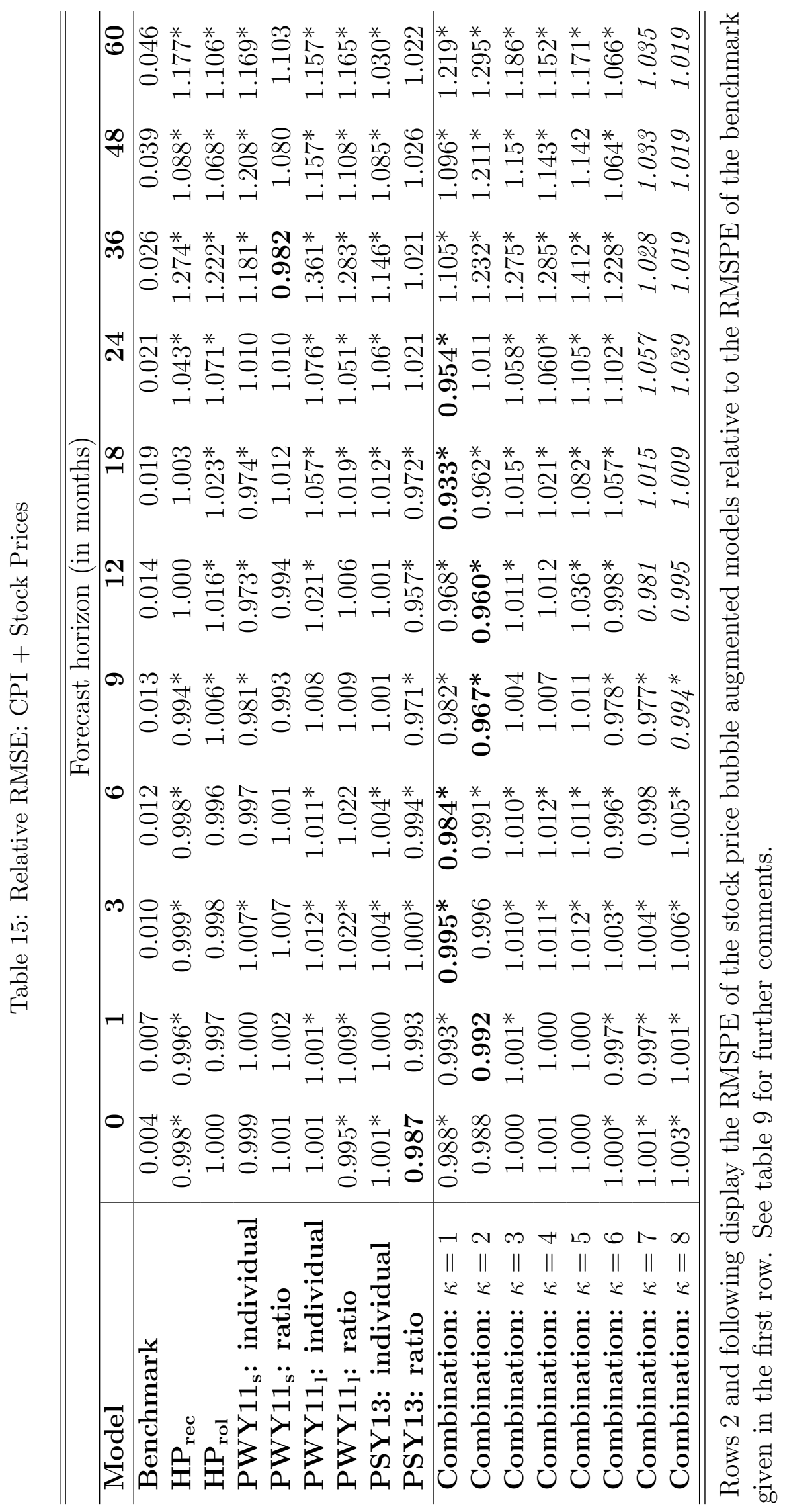




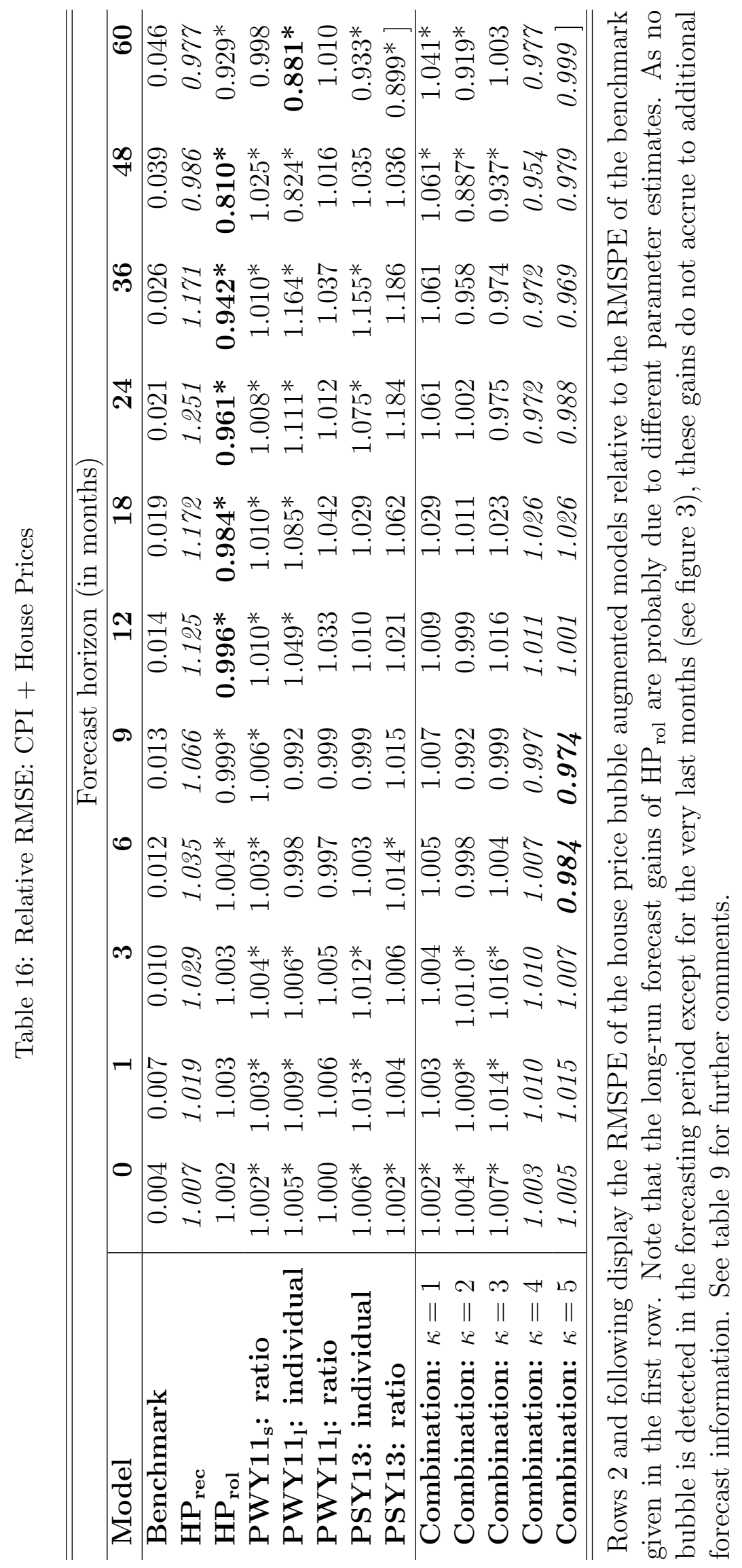




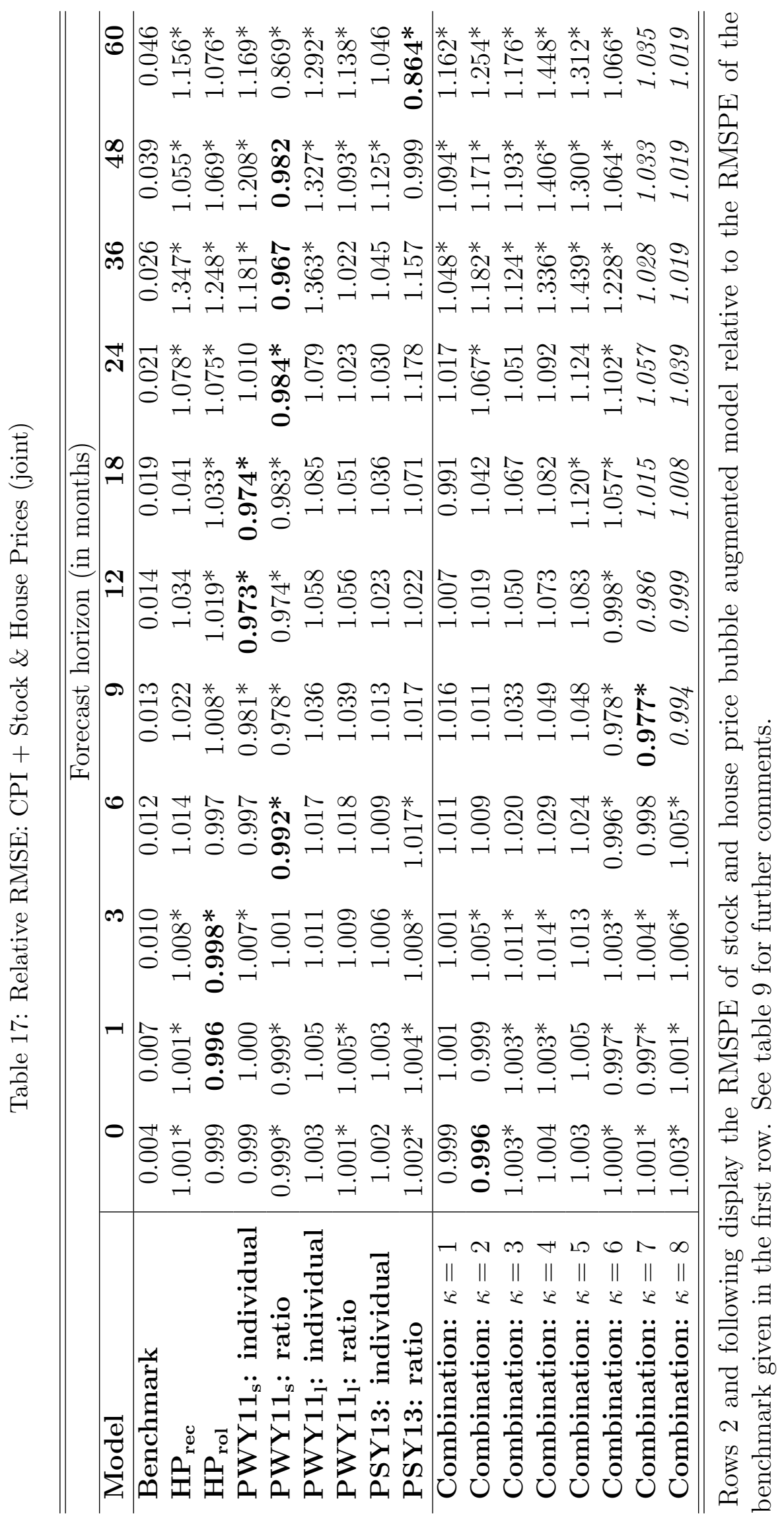

